\title{
Optical properties and crystal structure of triclinic growth sectors in vesuvianite
}

\author{
T. TANAKA*, M. AKIZUKı AND Y. KUDOH \\ Institute of Mincralogy, Petrology and Economic Geology, Faculty of Science, Tohoku University, Aoba-ku, Sendai \\ 980-8578, Japan
}

\section{ABSTRACT}

Sectored vesuvianite showing optically triclinic properties was studied by X-ray and P-FTIR analyses, and the origins of the internal optical texture are discussed. A monoclinic refinement (space group $P 2 / n$ ) suggests that site occupancies are slightly different among the $\mathrm{Al}(2)$ series, though the $\mathrm{OH}^{-}$dipole is randomly oriented in all sectors. A relationship between the surface and internal texture suggests that these sectoral structures were produced during crystal growth, not by phase transitions.

KeYwords: crystal structure, growth sectors, vesuvianite, crystal growth.

\section{Introduction}

Vesuvianite $\left(\mathrm{Ca}_{19}\left(\mathrm{Mg}, \mathrm{Fe}^{2+}, \mathrm{Al}, \mathrm{Fe}^{3+}\right)_{13} \mathrm{Si}_{18} \mathrm{O}_{68}\right.$ $\left.(\mathrm{O}, \mathrm{OH}, \mathrm{F})_{10}\right)$ is a widely-occurring rock-forming mineral (e.g. Johnson et al., 2000; Matsubara et al., 1998), but there remain certain problems related to its crystal structure, optical properties and chemical composition.

The space group of vesuvianite was first pronounced as $P 4 / n n c$ by Warren and Modell (1931), and this has been thought to be fundamental in the symmetry of vesuvianite (e.g. Lager et al., 1999). The crystal structure, however, has since been refined to space group $P 4 n c$ or $P 4 / n$ (e.g. Fitzgerald et al., 1986, 1987; Ohkawa et al., 1994; Armbruster and Gnos, 2000). Some vesuvianite crystals show weak reflections violating extinction rules on the $\langle 110\rangle$ glide plane. Groat et al. (1993) observed X-ray precession photographs parallel to the $b^{*}-c^{*}$ and $a^{*}-b^{*}$ planes, and found violating reflections suggesting $P 4 / n$ in some specimens with sectoral texture. Veblen and Wiechmann (1991) examined selected-area electron diffraction (SAED) patterns on $110^{*}$ and found numerous strong diffraction

* E-mail: t-tanaka@mail.cc.tohoku.ac.jp DOI: $10.1180 / 0026461026620027$

(c) 2002 The Mineralogical Society spots with $k+l=2 n+1$, which are consistent with violation of the $\{100\} n$-glide extinction rules in space group $P 4 / n n c$. They suggested that the violating diffractions are caused by the fine-scale domains, and that the space group is $P 2 / n$ or lower. Some vesuvianite crystals are optically biaxial, and therefore their structure must have lower than tetragonal symmetry.

Optical symmetry is lower than morphological symmetry in some minerals, and this phenomenon has been referred to as 'optical anomaly' since the $19^{\text {th }}$ century (Mallard, 1877). It has been suggested that the origin of these anomalies in many cases is crystal strain (e.g. Brauns, 1883). Although the morphological symmetry of vesuvianite is tetragonal, its optical anomaly has been known since the $19^{\text {th }}$ century (e.g. Brauns, 1891). The origin was explained as a strain or impurity in the crystal structure. Recently, the origin of the optical anomaly of vesuvianite was explained as psuedomerohedral twins of fine-scale domains resulting from a phase transition between a high-temperature $P 4 / n n c$ and a low-temperature $P 2 / n$ or lower structure (Veblen and Wiechmann, 1991). Groat et al. (1993) suggested that the origin of low symmetry is due to the ferroelastic phase transition, in which the space group is reduced from $P 4 / n n c$ to $P 4 / n$. On the other hand, Allen and Burnham (1992) suggested that the origin of the low optical symmetry is due to cation ordering during crystal 
growth. Thus, the origin of the optical anomaly of vesuvianite is not, as yet, definite.

The purpose of the present study is to analyse the crystal structure of optically triclinic vesuvianite, and discuss the origin of low-symmetry sectors.

\section{Experimental}

Optical observation

The specimens studied are from Bellecombe, Aosta, Italy. The crystal form observed by the scanning electron microscope is shown in Fig. 1. The crystal size is small $(\sim 1 \times 1 \times 5 \mathrm{~mm})$ and the crystal habit is variable. The colour is dark brown. The thin-sections parallel to the $(001)$ and $(110)$ faces are shown in Figs 2 and 4, respectively. This specimen is not altered, and the texture is clear

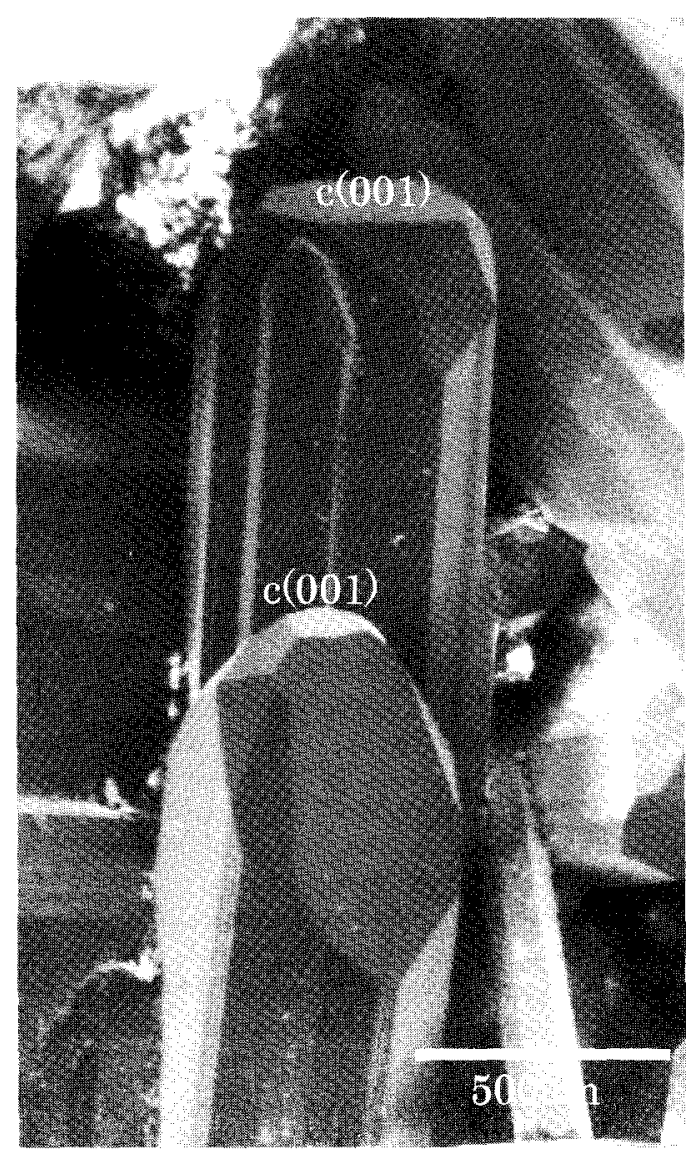

Flc. 1. SEM micrograph of vesuvianite from Bellecombe, Aosta, Italy. The $(001)$ face is small in the nearer crystal, but larger in that to the rear. under an optical microscope with crossed nicols. The specimens show optical growth sectors corresponding to the crystal faces. Some specimens from the same locality, however, are homogeneous when vicwed between crossed nicols. Figure 3 shows a schematic sketch of Fig. 2. Detailed optical observation suggests that the optical indicarix axes are not parallel to the morphological crystallographic axes in either the 110 ; or the $\{111\}$ sectors. In addition, the $\{110\}$ and $\{111\}$ sectors have several twins parallel to the (110) face, suggesting that the sectors are monoclinic or triclinic. In Fig. 4, the two black and white sectors related by twins correspond with the $\{111\}$ sector. The optic indicatrix axis, $X$, inclines at 3 to the $c$ axis, and therefore all optic indicatrix axes of the \{11\} sector are not parallel to the crystallographic axes, indicating optically triclinic symmetry. Furthermore, the $2 \mathrm{~V}$ value is larger in the $\{111\}$ sector $(2 \mathrm{~V} \alpha=45)$ than in the $110 ; \operatorname{sector}(2 \mathrm{~V} \alpha=$ 25 ). suggesting that the triclinicity of the $\{111\}$ sector is greater than that of the $\{110\}$ sector.

\section{Chemical composition}

The chemical composition was analysed by clectron microprobe analysis (JFOL-8800M) (Table 1), showing no significant difference betwcen the sectors. According to Groat et al. (1992), B-bearing vesuvianite is optically positive, and vesuvianite without $B$ is negative. The present specimens are optically negative, and therefore $B$ is assumed to be low in these specimens, though it was not analysed.

\section{Thermal and P-FTIR studies}

Although the specimens were heated up to $800 \mathrm{C}$, the sectors were still observed between crossed nicols, after cooling back to room temperature. The $2 \mathrm{~V}$ value of the scctors, however, became smaller after heating than before.

The triclinic sectors of the unheated specimens were observed by polarized Fourier Transform Infrared Spectroscopy (P-FTIR) (JEOL JIRDIAMOND-20 FTIR microspectrometer) in order to obtain the $\mathrm{OH}$-vibration spectra. The observation shows that the $\mathrm{OH}$ dipole is not in any preferred orientation in this specimen.

\section{Crystal structure refinement}

The single crystals were handpicked from the $\{110\}$ and $\{111\}$ sectors in the section parallel to 


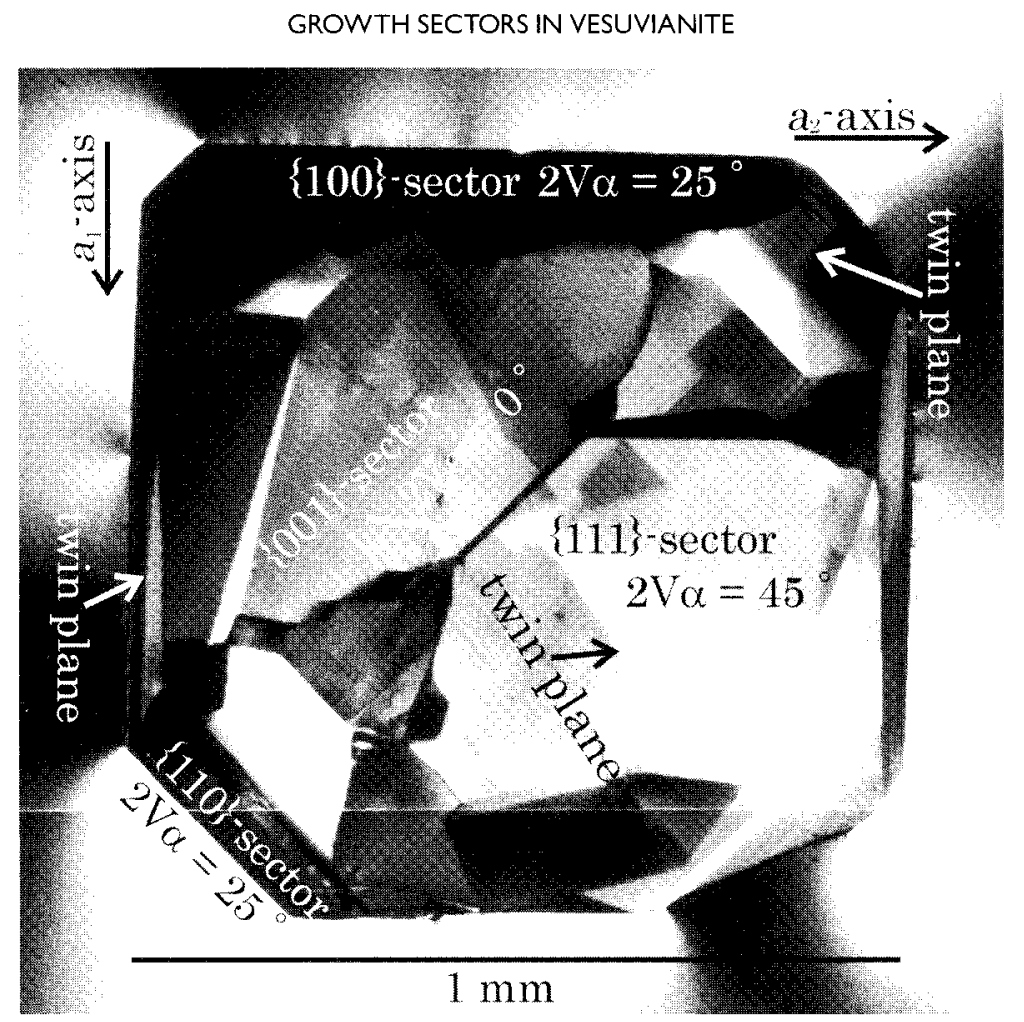

FIG. 2. The $\{001\},\{110\},\{111\}$ and other sectors with high index in the thin-section parallel to the (001) face. The $\{001\}$ sector is uniaxial, whereas the other sectors are biaxial $\left(2 \mathrm{~V} \alpha=25\right.$ in the $\{110\}$ sector, $2 \mathrm{~V} \alpha=45^{\circ}$ in the $\{111\}$ sector). Crossed nicols.

(001) face. The $\{100\}$ sector is small, and therefore the crystal could not be handpicked from this sector. The specimen of the $\{110\}$ sector used for the study is $70 \times 60 \times 60 \mu \mathrm{m}$ and the $\{111\}$ sector is $80 \times 70 \times 70 \mu \mathrm{m}$ in size. The lattice parameters were measured with a kappa four-circle diffractometer (MAC Science, MXC) with graphite-monochromated $\mathrm{Mo}-K \alpha$ radiation $(\lambda=0.71073 \AA)$ by a least-squares procedure applied to $\sin 2 \theta$ values of 48 reflections ranging from 30 to $35^{\circ}$ (Table 2). The precession photographs $\left(\mu=30^{\circ}, 72 \mathrm{~h}\right.$ exposure $)$ parallel to the $a^{*}-b^{*}, b^{*}-c^{*}$, and $110^{*}$ planes show no streaks or symmetry violating diffractions. In the lattice parameters of the $\{110\}$ and $\{111\}$ sectors, the $\beta$ angle is slightly larger than $90^{\circ}$, and the $\alpha, \gamma$ angles are $\sim 90$, indicating monoclinic symmetry.

The optic orientation shows that both the $\{110\}$ and $\{111\}$ sectors are triclinic, whereas the lattice parameters indicate monoclinic symmetry in these sectors. Since the triclinicity is low, the crystal structure refinements were carried out in monoclinic symmetry.

The linear absorption coefficient is $\sim 30.6 / \mathrm{cm}$, and therefore the difference of transmission factor between long and short lengths is $\sim 3 \%$, and can be ignored.

$X$-ray diffraction intensity data were measured at room temperature using the $\omega-20$ scan method from 3 to $60^{\circ}$ in the $\{110\}$ sector and from 3 to $55^{\circ}$ in the $\{111\}$ sector on an automated fourcircle single-crystal $\mathrm{X}$-ray diffractometer with graphite-monochromated Mo- $K \alpha_{1}$ radiation $(\lambda=$ $0.70930 \AA$ ). Scattering factors for all atoms were taken from the International Tables for $\mathrm{X}$-ray Crystallography (1974). After Lorentz and polarization corrections, sets of intensity data were obtained. No absorption corrections were made because of the small difference in transmission factor.

Since a Wilson plot showed the existence of an inversion centre in the structure, the space group 
T.TANAKA ETAL.

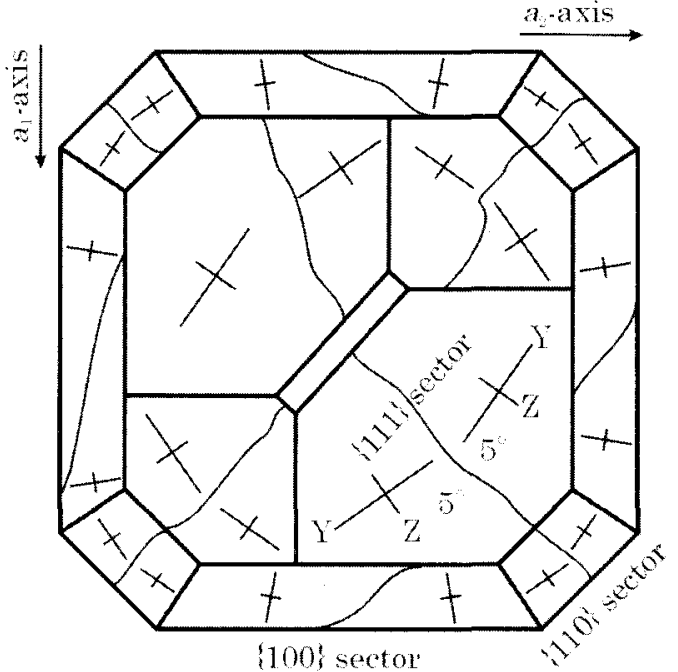

Fici. 3. Schematic internal texture in the (001) thin-section shown in Fig. 2. The $Z$ axis is nearly parallel to the morphological [100] direction in the $\{100\}$ sector, though that is nearly parallel to the morphological [110] direction in the $\{110\}$ and $\{111\}$ sectors. The angles between the $Z$ axis and morphological [110] direction are 5 in the $\{111\}$ and $\{110\}$ sectors and between the $Z$ axis and the [100] direction, 5 in the $\{100\}$ sectors also. Some curved lines show composition planes of twinning.

must correspond to $P 2 / n$, not $P n$. Therefore the crystal structures were refined using a full-matrix least-squares method in monoclinic $P 2 / n$.

The starting atomic coordinates for the monoclinic refinements were derived from the tetragonal structure given by Lager et al. (1999). All calculations were performed using the teXsan crystallographic software package of the Molecular Structure Corporation (1985, 1992). The results of the refinement are given in Table 3 .

The atomic coordinates and anisotropic displacement parameters are listed in Tables $4 a, 4 b, 5 a$
TABLE 1. Chemical analyses determined by EPMA.

\begin{tabular}{lrr}
\hline & $\{100\},\{110\}$ sector & $\{001\},\{111\}$ sector \\
\hline $\mathrm{SiO}_{2}$ & 37.13 & 37.35 \\
$\mathrm{TiO}_{2}$ & 1.09 & 1.21 \\
$\mathrm{Al}_{2} \mathrm{O}_{3}$ & 13.77 & 13.79 \\
$\mathrm{Fe}_{2} \mathrm{O}_{3}$ & 6.51 & 6.29 \\
$\mathrm{MnO}$ & 0.16 & 0.15 \\
$\mathrm{MgO}$ & 2.82 & 2.84 \\
$\mathrm{CaO}$ & 36.49 & 36.49 \\
$\mathrm{Na} 2$ & 0.00 & 0.00 \\
$\mathrm{~K}_{2} \mathrm{O}$ & 0.00 & 0.00 \\
$\mathrm{Si}$ & 18.00 & 18.00 \\
$\mathrm{Ti}$ & 0.40 & 0.44 \\
$\mathrm{Al}$ & 7.87 & 7.83 \\
$\mathrm{Fe}$ & 2.38 & 2.28 \\
$\mathrm{Mn}$ & 0.07 & 0.06 \\
$\mathrm{Mg}$ & 2.04 & 2.04 \\
$\mathrm{Ca}$ & 18.95 & 18.84 \\
$\mathrm{Na}$ & 0.00 & 0.00 \\
$\mathrm{~K}$ & 0.00 & 0.00 \\
\end{tabular}

Vesuvianite formula calculated on the basis of $18 \mathrm{Si}$

and $5 b$. Selected interatomic distances are summarized in Tables $6 a$ and $6 b$. There are slight differences between $\mathrm{Al}(2 \mathrm{a})-\mathrm{O}(2 \mathrm{a})$ and $\mathrm{Al}(2 \mathrm{~b})-\mathrm{O}(2 \mathrm{~b})$ and between $\mathrm{Al}(2 \mathrm{a})-\mathrm{O}(1 \mathrm{la})$ and $\mathrm{Al}(2 \mathrm{~b})-\mathrm{O}(1 \mathrm{lb})$ in the $\{110\}$ sector, and between $\mathrm{Al}(2 \mathrm{~b})-\mathrm{O}(2 \mathrm{~b})$ and $\mathrm{Al}(2 \mathrm{~d})-\mathrm{O}(2 \mathrm{~d})$ in the $\{111\}$ sector. Thus, both sectors are monoclinic.

The site occupancies of $\mathrm{Al}$ in the four $\mathrm{Al}(1)$ sites and the four $\mathrm{Al}(2)$ sites are shown in Table 7. Although the site occupancies of $\mathrm{Al}$ are similar among the four $\mathrm{Al}(1)$ sites in both the $\{110\}$ and $\{111\}$ sectors, the slight difference is observed at the four $A l(2)$ sites. The differences in site occupancies of the $A l(2)$ series are larger in the $\{111\}$ sector than in the $\{110\}$ sector.

TABLE 2. Lattice parameters of $\{110\}$ and $\{111\}$ sectors in vesuvianite.

\begin{tabular}{ccccccc}
\hline$\{110\}$ sector & & & & & & \\
$a$ & $b$ & $c$ & $\alpha$ & $\beta$ & $\gamma$ & $V_{\text {unit }}$ \\
$15.581(2)$ & $15.591(2)$ & $11.857(2)$ & $90.00(1)$ & $90.05(1)$ & $90.01(1)$ & $2880.31(60)$ \\
& & & & & & \\
$\{111\}$ sector & & & & & & \\
$a$ & $b$ & $c$ & $\alpha$ & $\beta$ & $\gamma$ & $V_{\text {unit }}$ \\
$15.582(2)$ & $15.586(2)$ & $11.855(1)$ & $89.99(1)$ & $90.05(1)$ & $89.99(1)$ & $2879.04(54)$
\end{tabular}

Using 48 reflections in the range $30.0<2 \theta<35.0$ 
GROWTH SECTORS IN VESUVIANITE

TABLE 3. Mode of data collection of vesuvianite.

\begin{tabular}{lll}
\hline Sector & $\{110\}$ sector & $\{111\}$ sector \\
\hline Dimension of crystal $\left(\mathrm{mm}^{3}\right)$ & $0.07 \times 0.06 \times 0.06$ & $0.08 \times 0.07 \times 0.07$ \\
Refinement space group & $P 2 / n$ & $P 2 / n$ \\
Scan type & $\omega-2 \theta$ & $\omega-2 \theta$ \\
Max. 20 & 60.0 & 55.0 \\
Reflections measured & 17312 & 7209 \\
Unique reflection & 9355 & 7020 \\
Reflections used in the refinement $\left(I>3 \sigma_{I}\right)$ & 5705 & 4602 \\
R value $(\%)$ & 6.9 & 6.8 \\
Rw value $(\%)^{*}$ & 5.0 & 5.6 \\
GoF & 1.42 & 1.85 \\
& &
\end{tabular}

$*_{\mathrm{w}}=1 / \sigma^{2}$

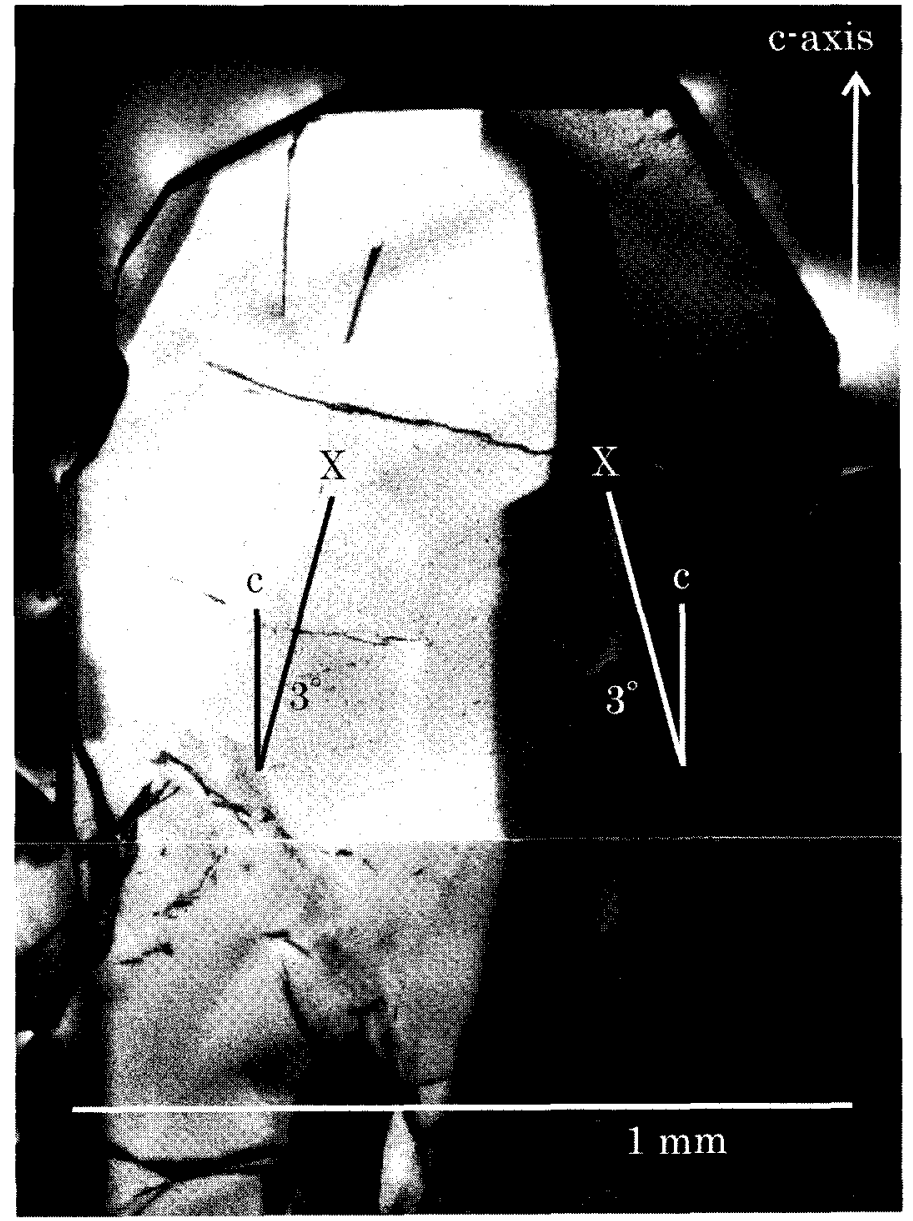

FIG. 4. Polarized photograph showing the $\{111\}$ growth sector in a section parallel to (110). The optic indicatrix axis $X$ inclines symmetrically at 3 to the $c$ axis in the two black and white sectors, which are related by twinning. Crossed nicols. 


\section{Discussion}

\section{Optical observation and crystal structure}

The specimen is optically biaxial, and the symmetry is triclinic, though the triclinicity is low. Tetragonal vesuvianite shows two kinds of diads: one is parallel to [100] and the other is parallel to $[110]$. Figure 3 shows that the optical indicatrix axis $Z$ is approximately parallel to the [100] direction in the $\{100\}$ sector, and is approximately parallel to the [110] direction in the both $\{110\}$ and $\{11\}\}$ sectors. The monoclinic diad obtained by XRD corresponds to the $Z$ direction, i.e. the crystal orientations are different in different sectors.

Since the first crystal structure analysis of Warren and Modell (1931), several different models have been proposed for the structure of vesuvianite. Although the space group was assumed to be P4/nnc by Warren and Modell (1931), Armbruster and Gnos (2000) refined the crystal structure to space groups $P 4 n c$ and $P 4 / n$. The 4- and $\overline{4}$-fold axes, however, were not observed in the present specimens. Since the specimen is optically triclinic, the correct structural symmetry must be triclinic, even if the triclinicity is low.

The differences of Al occupancy between Al(2a) and $\mathrm{Al}(2 \mathrm{~b})$, and $\mathrm{Al}(2 \mathrm{c})$ and $\mathrm{Al}(2 \mathrm{~d})$ are 5.8 and 4.4 times standard deviation in the $\{110\}$ sector, respectively. Also, the difference is 9.0 and 5.3 times in the $\{111\}$ sector, respectively. Therefore the symmetry is reduced from tetragonal to at least monoclinic in both the $\{110\}$ and $\{111\}$ sectors. Furthermore, the difference in the Al occupancy in the $\{111\}$ sector $\left(2 \mathrm{~V} \alpha=45^{\circ}\right)$ is larger than that in the $\{110\}$ sector $\left(2 \mathrm{~V} \alpha=25^{\circ}\right)$ (Fig. 5). It is suggested that the triclinicity is greater in the $\{111\}$ sector than in the $\{110\}$ sector. X-ray analysis correlates with the optical observation.

\section{Origin of sectoral structure}

Similar sectors are obscrved in adularia (Akizuki and Sunagawa, 1978), topaz (Akizuki et al., 1979), grossular-andradite garnet (Akizuki, 1984; Shtukenberg et al., 2001), apophyllite (Akizuki and Terada, 1998) and some other minerals (e.g. alum in Shtukenberg et al., 2000). It is thought that the sectors result from cation $\left(\mathrm{Al}^{3+} / \mathrm{Si}^{4+}, \mathrm{Al}^{3+} / \mathrm{Fe}^{3+}\right)$ or anion $\left(\mathrm{OH}^{-} / \mathrm{F}^{-}\right)$ ordering or preferred orientation of the $\mathrm{OH}^{-}$ dipole during crystal growth.

Akizuki et al. (1979) suggested that the sectoral textures of topaz become extinct upon heating, and Akizuki and Terada (1998) suggested that the low symmetry of apophyllite is due to preferred orientation of the $\mathrm{OH}^{-}$dipole. Groat et al. (1993) found that the sectors of vesuvianite are extinct at $500 \mathrm{C}$, and Groat et al. (1995) suggested the substitution of $\mathrm{H}$ and $\mathrm{B}$ atoms, after interpretation of the IR spectra in the $\mathrm{OH}$ region of tetragonal
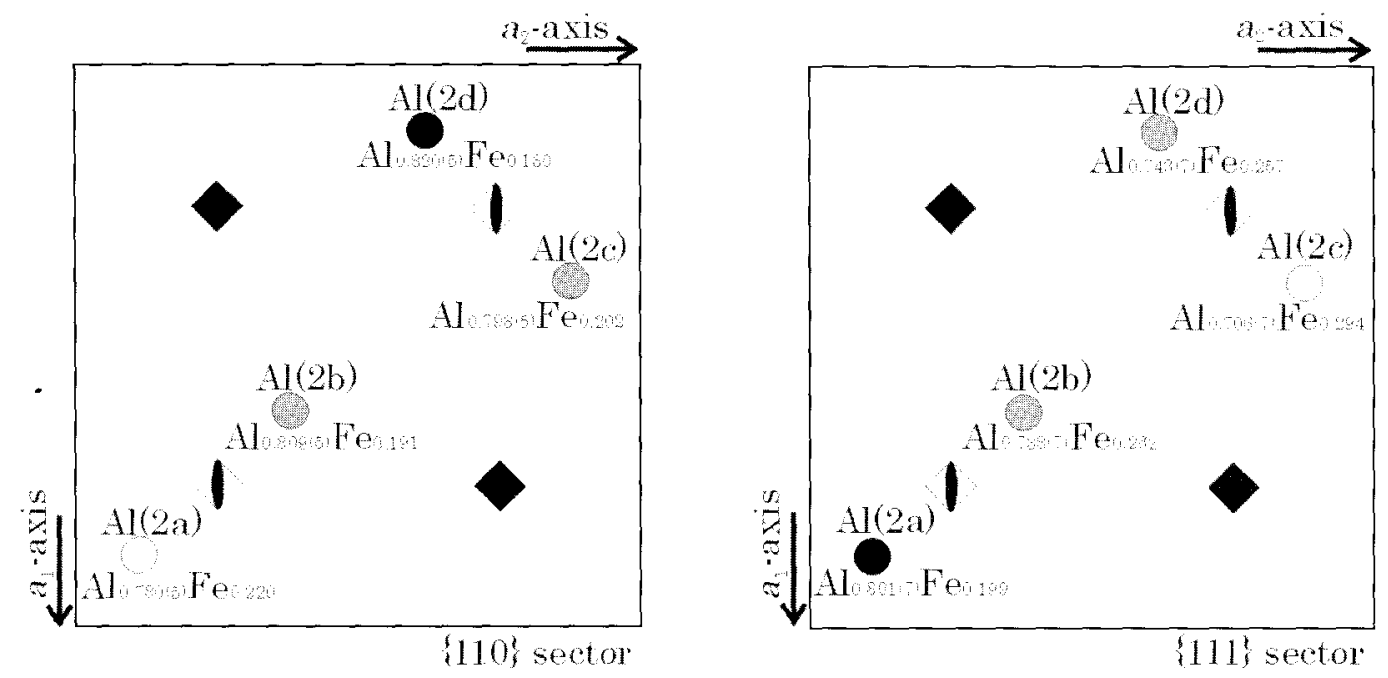

FlG. 5. Al site occupancies of $\mathrm{Al}(2)$ series around the $\overline{4}$-fold axis in $\{110\}$ and $\{111\}$ sectors. The difference of $\mathrm{Al}$ population in the $\{111\}$ sector is larger than that in the $\{110\}$ sector. 
GROWTH SECTORS IN VESUVIANITE

TABLE $4 a$. Atomic coordinates of $\{110\}$ sector in vesuvianite.

\begin{tabular}{|c|c|c|c|c|c|c|c|c|c|}
\hline Atom & $x$ & $y$ & $\bar{z}$ & $B_{\mathrm{eq}}\left(\AA^{2}\right)$ & Atom & $x$ & $y$ & $z$ & $B_{\text {eq }}\left(\AA^{2}\right)$ \\
\hline $\mathrm{Ca}$ & $-0.2498(2)$ & $-0.2499(2)$ & $0.1493(2)$ & $0.53(4)$ & $O(2 b)$ & $0.6172(3)$ & $0.3392(3)$ & $0.2805(4)$ & $0.46(8)$ \\
\hline $\mathrm{Ca}(\mathrm{la})$ & -0.2500 & $0.2502(1)$ & 0.2500 & $0.39(3)$ & $O(2 c)$ & $0.3393(3)$ & $-0.1174(3)$ & $0.2794(4)$ & $0.43(8)$ \\
\hline $\mathrm{Ca}(1 \mathrm{~b})$ & 0.2500 & $-0.2500(1)$ & 0.2500 & $0.40(3)$ & $O(2 d)$ & $0.1604(3)$ & $0.6170(3)$ & $0.2800(4)$ & $0.42(8)$ \\
\hline $\mathrm{Ca}(2 a)$ & $-0.18914(8)$ & $0.04437(9)$ & $0.3797(1)$ & $0.41(2)$ & $O(3 a)$ & $-0.0473(3)$ & $0.2227(3)$ & $0.0734(4)$ & $0.49(8)$ \\
\hline $\mathrm{Ca}(2 \mathrm{~b})$ & $0.68921(8)$ & $0.45565(9)$ & $0.3796(1)$ & $0.36(2)$ & $O(3 b)$ & $0.5471(3)$ & $0.2769(3)$ & $0.0733(4)$ & $0.35(8)$ \\
\hline $\mathrm{Ca}(2 \mathrm{c})$ & $0.45594(9)$ & $-0.18920(8)$ & $0.3794(1)$ & $0.40(2)$ & $O(3 c)$ & $0.2772(3)$ & $-0.0473(3)$ & $0.0741(4)$ & $0.48(8)$ \\
\hline $\mathrm{Ca}(2 \mathrm{~d})$ & $0.04416(9)$ & $0.68911(8)$ & $0.3799(1)$ & $0.40(2)$ & $O(3 d)$ & $0.2228(3)$ & $0.5474(3)$ & $0.0739(4)$ & $0.41(8)$ \\
\hline $\mathrm{Ca}(3 \mathrm{a})$ & $-0.10136(9)$ & $-0.18173(10)$ & $0.8882(1)$ & $1.13(3)$ & $O(4 a)$ & $-0.0612(3)$ & $0.1055(3)$ & $0.4701(4)$ & $0.51(8)$ \\
\hline $\mathrm{Ca}(3 \mathrm{~b})$ & $0.60113(9)$ & $0.68176(9)$ & $0.8878(1)$ & $0.99(3)$ & $O(4 b)$ & $0.5606(3)$ & $0.3942(3)$ & $0.4707(4)$ & $0.49(8)$ \\
\hline $\mathrm{Ca}(3 \mathrm{c})$ & $0.68183(9)$ & $-0.10125(9)$ & $0.8880(1)$ & $1.03(3)$ & $O(4 c)$ & $0.3938(3)$ & $-0.0609(3)$ & $0.4694(4)$ & $0.50(8)$ \\
\hline $\mathrm{Ca}(3 \mathrm{~d})$ & $-0.18165(9)$ & $0.60127(9)$ & $0.8882(1)$ & $1.10(3)$ & $O(4 d)$ & $0.1065(3)$ & $0.5615(3)$ & $0.4693(4)$ & $0.46(8)$ \\
\hline $\operatorname{Si}(1)$ & $-0.2499(1)$ & $0.2500(1)$ & $0.0000(1)$ & $0.29(3)$ & $O(5 a)$ & $-0.1705(3)$ & $0.0133(3)$ & $0.1802(4)$ & $0.61(9)$ \\
\hline $\operatorname{Si}(2 a)$ & $-0.1803(1)$ & $0.0402(1)$ & $0.8717(1)$ & $0.29(3)$ & $O(5 b)$ & $0.6711(3)$ & $0.4865(3)$ & $0.1804(4)$ & $0.59(9)$ \\
\hline $\mathrm{Si}(2 b)$ & $0.6805(1)$ & $0.4600(1)$ & $0.8714(1)$ & $0.31(3)$ & $O(5 c)$ & $0.4867(3)$ & $-0.1710(3)$ & $0.1803(4)$ & $0.56(8)$ \\
\hline $\operatorname{Si}(2 \mathrm{c})$ & $0.4596(1)$ & $-0.1805(1)$ & $0.8715(1)$ & $0.26(3)$ & $O(5 d)$ & $0.0135(3)$ & $0.6700(3)$ & $0.1797(4)$ & $0.70(9)$ \\
\hline $\operatorname{Si}(2 d)$ & $0.0402(1)$ & $0.6803(1)$ & $0.8714(1)$ & $0.24(3)$ & $O(6 a)$ & $-0.1203(3)$ & $-0.2722(3)$ & $0.0588(4)$ & $0.76(9)$ \\
\hline $\mathrm{Si}(3 \mathrm{a})$ & $-0.0839(1)$ & $-0.1506(1)$ & $0.3641(1)$ & $0.34(3)$ & $O(6 b)$ & $0.6202(3)$ & $0.7719(3)$ & $0.0586(4)$ & $0.91(9)$ \\
\hline $\operatorname{Si}(3 b)$ & $0.5837(1)$ & $0.6507(1)$ & $0.3642(1)$ & $0.34(3)$ & $O(60)$ & $0.7722(3)$ & $-0.1201(3)$ & $0.0581(4)$ & $0.84(9)$ \\
\hline $\operatorname{Si}(30)$ & $0.6508(1)$ & $-0.0838(1)$ & $0.3641(1)$ & $0.31(3)$ & $O(6 \mathrm{~d})$ & $-0.2719(3)$ & $0.6206(3)$ & $0.0588(4)$ & $0.81(9)$ \\
\hline $\operatorname{Si}(3 d)$ & $-0.1508(1)$ & $0.5838(1)$ & $0.3643(1)$ & $0.36(3)$ & & $0.0556(3)$ & $0.1730(3)$ & $0.3202(4)$ & $0.61(9)$ \\
\hline $\mathrm{Al}(\mathrm{la})$ & 0.0000 & 0.0000 & 0.0000 & $0.40(5)$ & $O(7 b)$ & $0.4443(3)$ & $0.3273(3)$ & $0.3204(4)$ & $0.65(9)$ \\
\hline $\mathrm{Al}(\mathrm{lb})$ & 0.5000 & 0.5000 & 0.0000 & $0.44(5)$ & $O(70)$ & $0.3270(3)$ & $0.0554(3)$ & $0.3207(4)$ & $0.70(9)$ \\
\hline $\mathrm{Al}(\mid \mathrm{c})$ & 0.5000 & 0.0000 & 0.0000 & $0.46(5)$ & $O(7 d)$ & $0.1727(3)$ & $0.4445(3)$ & $0.3203(4)$ & $0.68(9)$ \\
\hline $\operatorname{Al}(\mathrm{ld})$ & 0.0000 & 0.5000 & 0.0000 & $0.51(5)$ & $O(8 a)$ & $-0.0607(3)$ & $-0.0914(3)$ & $0.0660(4)$ & $0.50(8)$ \\
\hline $\operatorname{Al}(2 a)$ & $-0.1119(1)$ & $0.1213(1)$ & $0.1269(1)$ & $0.42(4)$ & $O(8 b)$ & $0.5611(3)$ & $0.5909(3)$ & $0.0654(4)$ & $0.51(8)$ \\
\hline $\mathrm{Al}(2 \mathrm{~b})$ & $0.6118(1)$ & $0.3785(1)$ & $0.1270(1)$ & $0.43(4)$ & $O(8 c)$ & $0.5913(3)$ & $-0.0612(3)$ & $0.0665(4)$ & $0.44(8)$ \\
\hline $\mathrm{Al}(2 \mathrm{c})$ & $0.3787(1)$ & $-0.1119(1)$ & $0.1266(1)$ & $0.47(4)$ & $O(8 \mathrm{~d})$ & $-0.0910(3)$ & $0.5609(3)$ & $0.0662(4)$ & $0.52(8)$ \\
\hline $\operatorname{Al}(2 \mathrm{~d})$ & $0.1213(1)$ & $0.6120(1)$ & $0.1268(1)$ & $0.32(4)$ & $O(9 a)$ & $-0.1452(3)$ & $-0.1452(3)$ & $0.2501(4)$ & $0.62(9)$ \\
\hline $\mathrm{Mg}$ & $-0.2497(1)$ & $-0.2499(1)$ & $0.0356(2)$ & $0.78(4)$ & $O(9 b)$ & $0.6451(3)$ & $0.6459(3)$ & $0.2499(4)$ & $0.61(9)$ \\
\hline$O($ la) & $-0.2207(3)$ & $0.1728(3)$ & $0.0838(4)$ & $0.53(8)$ & $O(10)$ & $-0.2499(3)$ & $-0.2497(4)$ & $0.8650(5)$ & $1.28(10)$ \\
\hline$O(I b)$ & $0.7215(3)$ & $0.3270(3)$ & $0.0841(4)$ & $0.46(8)$ & $O(11 a)$ & $-0.0037(3)$ & $0.0617(3)$ & $0.1364(4)$ & $0.70(9)$ \\
\hline$O(1 c)$ & $0.3270(3)$ & $-0.2209(3)$ & $0.0835(4)$ & $0.52(8)$ & $O(11 b)$ & $0.5025(3)$ & $0.4390(3)$ & $0.1362(4)$ & $0.68(9)$ \\
\hline$O(1 d)$ & $0.1729(3)$ & $0.7209(3)$ & $0.0839(4)$ & $0.45(8)$ & $O(11 c)$ & $0.4386(3)$ & $-0.0031(3)$ & $0.1361(4)$ & $0.56(8)$ \\
\hline$O(2 a)$ & $-0.1168(3)$ & $0.1603(3)$ & $0.2791(4)$ & $0.51(8)$ & $O(11 d)$ & $0.0615(3)$ & $0.5032(3)$ & $0.1362(4)$ & $0.69(9)$ \\
\hline
\end{tabular}

Table $4 b$. Atomic coordinates of $\{111\}$ sector in vesuvianite.

\begin{tabular}{|c|c|c|c|c|c|c|c|c|c|}
\hline Atom & $x$ & $y$ & $z$ & $B_{\mathrm{eq}}\left(\AA^{2}\right)$ & Atom & $x$ & $y$ & $z$ & $B_{\text {cl }}\left(\AA^{2}\right.$ \\
\hline $\mathrm{Ca}$ & $-0.2504(3)$ & $-0.2493(3)$ & $0.1472(4)$ & $1.00(8)$ & $A l(1 b)$ & 0.5000 & 0.5000 & 0.0000 & $0.38(9)$ \\
\hline $\mathrm{Ca}(\mathrm{la})$ & -0.2500 & $0.2496(2)$ & 0.2500 & $0.44(5)$ & $A(1 c)$ & 0.5000 & 0.0000 & 0.0000 & $0.59(10)$ \\
\hline $\mathrm{Ca}(1 \mathrm{~b})$ & 0.2500 & $-0.2502(3)$ & 0.2500 & $0.54(6)$ & All ld) & 0.0000 & 0.5000 & 0.0000 & $0.41(9)$ \\
\hline $\operatorname{Ca}(2 a)$ & $-0.1893(1)$ & $0.0443(2)$ & $0.3794(2)$ & $0.50(4)$ & $\mathrm{Al}(2 \mathrm{a})$ & $-0.1119(2)$ & $0.1215(2)$ & $0.1272(2)$ & $0.99(9)$ \\
\hline $\mathrm{Ca}(2 \mathrm{~b})$ & $0.6891(1)$ & $0.4558(2)$ & $0.3797(2)$ & $0.45(4)$ & $\Lambda \mathrm{l}(2 \mathrm{~b})$ & $0.6117(2)$ & $0.3787(2)$ & $0.1269(2)$ & $0.98(10)$ \\
\hline $\mathrm{Ca}(2 \mathrm{c})$ & $0.4554(1)$ & $-0.1890(2)$ & $0.3800(2)$ & $0.44(4)$ & $\mathrm{Al}(2 \mathrm{c})$ & $0.3786(2)$ & $-0.1115(2)$ & $0.1270(2)$ & $1.6(1)$ \\
\hline $\mathrm{Ca}(2 \mathrm{~d})$ & $0.0440(1)$ & $0.6892(2)$ & $0.3793(2)$ & $0.46(4)$ & $\mathrm{Al}(2 \mathrm{~d})$ & $0.1211(2)$ & $0.6119(2)$ & $0.1267(2)$ & $1.2(1)$ \\
\hline $\mathrm{Ca}(3 \mathrm{a})$ & $-0.1010(1)$ & $-0.1816(2)$ & $0.8882(2)$ & $0.93(5)$ & $\mathrm{Mg}$ & $-0.2502(2)$ & $-0.2497(3)$ & $0.0363(3)$ & $1.30(9)$ \\
\hline $\mathrm{Ca}(3 \mathrm{~b})$ & $0.6012(2)$ & $0.6819(2)$ & $0.8879(2)$ & $0.97(5)$ & $O(1 a)$ & $-0.2221(5)$ & $0.1725(5)$ & $0.0853(6)$ & $0.6(2)$ \\
\hline$(a(3 c)$ & $0.6818(2)$ & $-0.1011(2)$ & $0.8882(2)$ & $0.97(5)$ & $O(1 b)$ & $0.7216(5)$ & $0.3280(5)$ & $0.0847(6)$ & $0.3(1)$ \\
\hline $\mathrm{Ca}(3 d)$ & $-0.1817(2)$ & $0.6012(2)$ & $0.8885(2)$ & $0.98(5)$ & $O(l c)$ & $0.3278(5)$ & $-0.2214(5)$ & $0.0852(6)$ & $0.5(1)$ \\
\hline $\operatorname{Si}(1)$ & $-0.2503(2)$ & $0.2501(2)$ & $0.0002(2)$ & $0.25(5)$ & $O(I d)$ & $0.1731(5)$ & $0.7218(5)$ & $0.0840(6)$ & $0.6(1)$ \\
\hline $\operatorname{Si}(2 \mathrm{a})$ & $-0.1804(2)$ & $0.0402(2)$ & $0.8714(3)$ & $0.41(6)$ & $O(2 a)$ & $-0.1175(5)$ & $0.1609(5)$ & $0.2804(6)$ & $0.7(2)$ \\
\hline $\operatorname{Si}(2 b)$ & $0.6804(2)$ & $0.4599(2)$ & $0.8716(3)$ & $0.29(6)$ & $O(2 b)$ & $0.6169(1)$ & $0.3399(5)$ & $0.2787(6)$ & $0.6(2)$ \\
\hline $\operatorname{Si}(2 c)$ & $0.4595(2)$ & $-0.1803(2)$ & $0.8718(3)$ & $0.34(6)$ & $O(2 c)$ & $0.3386(5)$ & $0.1173(5)$ & $0.2794(6)$ & $0.6(2)$ \\
\hline $\operatorname{Si}(2 d)$ & $0.0398(2)$ & $0.6802(2)$ & $0.8716(3)$ & $0.40(6)$ & $O(2 d)$ & $0.1606(5)$ & $0.6171(5)$ & $0.2807(6)$ & $0.6(2)$ \\
\hline $\operatorname{Si}(3 a)$ & $-0.0836(2)$ & $0.1504(2)$ & $0.3637(3)$ & $0.39(6)$ & $O(3 a)$ & $-0.0460(5)$ & $0.2235(5)$ & $0.0747(6)$ & $0.5(1)$ \\
\hline $\operatorname{Si}(3 b)$ & $0.5839(2)$ & $0.6503(2)$ & $0.3641(3)$ & $0.46(6)$ & $O(3 b)$ & $0.5473(5)$ & $0.2773(5)$ & $0.0745(6)$ & $0.5(1)$ \\
\hline $\operatorname{Si}(3 c)$ & $0.6505(2)$ & $-0.0837(2)$ & $0.3643(3)$ & $0.47(6)$ & $O(3 c)$ & $0.2771(5)$ & $-0.0463(5)$ & $0.0750(6)$ & $0.6(2)$ \\
\hline $\operatorname{Si}(3 d)$ & $-0.1502(2)$ & $0.5839(2)$ & $0.3648(3)$ & $0.42(6)$ & $O(3 d)$ & $0.2234(5)$ & $0.5469(5)$ & $0.0739(6)$ & $0.6(2)$ \\
\hline $\mathrm{N}(\mathrm{la})$ & 0.0000 & 0.0000 & 0.0000 & $0.38(9)$ & $O(4 a)$ & $-0.0615(4)$ & $0.1061(5)$ & $0.4692(6)$ & $0.3(1)$ \\
\hline
\end{tabular}


T.TANAKA ETAL.

Table $4 b$. (contd.) Atomic coordinates of $\{111\}$ sector in vesuvianite.

\begin{tabular}{|c|c|c|c|c|c|c|c|c|c|}
\hline Atom & $x$ & $y$ & $z$ & $B_{\mathrm{eq}}\left(\AA^{2}\right)$ & Atom & $x$ & $y$ & $z$ & $B_{\mathrm{eq}}\left(\AA^{2}\right)$ \\
\hline$O(4 b)$ & $0.5617(5)$ & $0.3937(5)$ & $0.4688(6)$ & $0.6(2)$ & $O(7 c)$ & $0.3272(5)$ & $0.0560(5)$ & $0.3232(7)$ & $0.7(2)$ \\
\hline$O(4 c)$ & $0.3939(4)$ & $-0.0612(5)$ & $0.4694(6)$ & $0.7(2)$ & $O(7 \mathrm{~d})$ & $0.1722(5)$ & $0.4435(5)$ & $0.3231(7)$ & $0.7(2)$ \\
\hline$O(4 d)$ & $0.1062(5)$ & $0.5610(5)$ & $0.4693(6)$ & $0.5(2)$ & $O(8 a)$ & $-0.0612(5)$ & $-0.0919(5)$ & $0.0667(7)$ & $0.6(2)$ \\
\hline$O(5 a)$ & $-0.1709(5)$ & $0.0132(5)$ & $0.1807(6)$ & $0.7(2)$ & $O(8 b)$ & $0.5613(5)$ & $0.5911(5)$ & $0.0667(7)$ & $0.8(2)$ \\
\hline $0(5 b)$ & $0.6701(5)$ & $0.4866(5)$ & $0.1800(6)$ & $0.7(2)$ & $O(8 c)$ & $0.5923(5)$ & $-0.0607(5)$ & $0.0666(7)$ & $0.7(2)$ \\
\hline$O(5 c)$ & $0.4866(5)$ & $-0.1702(5)$ & $0.1810(7)$ & $0.8(2)$ & $O(8 d)$ & $-0.0910(5)$ & $0.5615(5)$ & $0.0657(7)$ & $0.8(2)$ \\
\hline$O(5 d)$ & $0.0127(5)$ & $0.6714(5)$ & $0.1793(6)$ & $0.7(2)$ & $O(9 a)$ & $-0.1450(5)$ & $-0.1454(6)$ & $0.2499(7)$ & $0.9(2)$ \\
\hline$O(6 a)$ & $0.1212(5)$ & $-0.2728(5)$ & $0.0600(6)$ & $0.9(2)$ & $O(9 b)$ & $0.6449(5)$ & $0.6454(6)$ & $0.2503(6)$ & $0.9(2)$ \\
\hline$O(6 b)$ & $0.6203(5)$ & $0.7724(5)$ & $0.0592(6)$ & $0.9(2)$ & $O(10)$ & $-0.2500(5)$ & $-0.2495(7)$ & $0.8617(7)$ & $1.6(2)$ \\
\hline$O(6 c)$ & $0.7710(5)$ & $-0.1209(5)$ & $0.0595(6)$ & $0.8(2)$ & $O(1 / a)$ & $-0.0033(5)$ & $0.0613(5)$ & $0.1352(7)$ & $0.8(2)$ \\
\hline$O(6 \mathrm{~d})$ & $-0.2727(5)$ & $0.6206(5)$ & $0.0601(7)$ & $0.9(2)$ & $O(1 \mid b)$ & $0.5032(5)$ & $0.4387(5)$ & $0.1349(6)$ & $0.7(2)$ \\
\hline$O(7 a)$ & $0.0554(5)$ & $0.1731(5)$ & $0.3233(6)$ & $0.8(2)$ & $O(11 c)$ & $0.4393(5)$ & $-0.0037(5)$ & $0.1334(6)$ & $0.8(2)$ \\
\hline$O(7 b)$ & $0.4442(5)$ & $0.3276(5)$ & $0.3228(7)$ & $0.9(2)$ & $O(11 d)$ & $0.0612(5)$ & $0.5035(5)$ & $0.1346(6)$ & $0.7(2)$ \\
\hline
\end{tabular}

TABLI: $5 a$. Anisotropic displacement parameters of $\{110\}$ sector.

\begin{tabular}{|c|c|c|c|c|c|c|}
\hline Atom & $U_{11}$ & $U_{22}$ & $U_{33}$ & $U_{12}$ & $U_{13}$ & $U_{23}$ \\
\hline $\mathrm{Ca}$ & $0.004(1)$ & $0.0010(10)$ & $0.015(1)$ & $-0.0005(9)$ & $0.0014(9)$ & $0.0001(10)$ \\
\hline $\mathrm{Ca}(1 \mathrm{a})$ & $0.0099(8)$ & $0.0030(7)$ & $0.0021(7)$ & 0.0000 & $-0.0008(6)$ & 0.0000 \\
\hline $\mathrm{Ca}(1 \mathrm{~b})$ & $0.0031(7)$ & $0.0106(8)$ & $0.0016(7)$ & 0.0000 & $0.0005(6)$ & 0.0000 \\
\hline $\mathrm{Ca}(2 \mathrm{a})$ & $0.0032(6)$ & $0.0068(6)$ & $0.0055(6)$ & $0.0010(5)$ & $-0.0002(4)$ & $0.0010(4)$ \\
\hline $\mathrm{Ca}(2 \mathrm{~b})$ & $0.0039(6)$ & $0.0045(6)$ & $0.0053(6)$ & $0.0009(4)$ & $0.0013(4)$ & $-0.0010(4)$ \\
\hline $\mathrm{Ca}(2 \mathrm{c})$ & $0.0065(6)$ & $0.0039(6)$ & $0.0049(6)$ & $-0.0005(4)$ & $-0.0003(4)$ & $-0.0002(4)$ \\
\hline $\mathrm{Ca}(2 \mathrm{~d})$ & $0.0055(6)$ & $0.0040(6)$ & $0.0056(6)$ & $-0.0006(4)$ & $0.0009(4)$ & $0.0003(4)$ \\
\hline $\mathrm{Ca}(3 \mathrm{a})$ & $0.0084(6)$ & $0.0106(7)$ & $0.0240(8)$ & $0.0034(5)$ & $-0.0078(6)$ & $-0.006 l(6)$ \\
\hline $\mathrm{Ca}(3 \mathrm{~b})$ & $0.0083(6)$ & $0.0079(6)$ & $0.0215(8)$ & $0.0027(5)$ & $0.0071(5)$ & $0.0065(5)$ \\
\hline $\mathrm{Ca}(3 \mathrm{c})$ & $0.0090(7)$ & $0.0085(6)$ & $0.0214(8)$ & $-0.0037(5)$ & $0.0078(5)$ & $-0.0081(5)$ \\
\hline $\mathrm{Ca}(3 \mathrm{~d})$ & $0.0092(7)$ & $0.0084(6)$ & $0.0241(8)$ & $-0.0037(5)$ & $-0.0070(5)$ & $0.0093(6)$ \\
\hline Si(1) & $0.0060(7)$ & $0.0040(7)$ & $0.0009(7)$ & $-0.0003(6)$ & $0.0008(5)$ & $-0.0010(6)$ \\
\hline $\operatorname{Si}(2 a)$ & $0.0029(8)$ & $0.0042(8)$ & $0.0037(8)$ & $0.0003(6)$ & $0.0006(6)$ & $0.0014(6)$ \\
\hline $\operatorname{Si}(2 b)$ & $0.0034(8)$ & $0.0035(8)$ & $0.0049(8)$ & $-0.0004(6)$ & $0.0003(6)$ & $-0.0011(6)$ \\
\hline $\operatorname{Si}(2 c)$ & $0.0031(8)$ & $0.0029(7)$ & $0.0039(8)$ & $0.0007(6)$ & $-0.0008(6)$ & $-0.0004(6)$ \\
\hline $\operatorname{Si}(2 d)$ & $0.0026(8)$ & $0.0027(8)$ & $0.0040(8)$ & $-0.0002(6)$ & $0.0016(6)$ & $-0.0001(6)$ \\
\hline $\operatorname{Si}(3 a)$ & $0.0081(8)$ & $0.0016(7)$ & $0.0031(8)$ & $0.0006(6)$ & $0.0001(6)$ & $0.0004(6)$ \\
\hline $\operatorname{Si}(3 b)$ & $0.0077(8)$ & $0.0013(7)$ & $0.0037(8)$ & $0.0000(6)$ & $0.0006(6)$ & $0.0005(6)$ \\
\hline $\operatorname{Si}(3 c)$ & $0.0013(7)$ & $0.0065(8)$ & $0.0040(8)$ & $0.0002(6)$ & $0.0000(6)$ & $-0.0004(6)$ \\
\hline $\operatorname{Si}(3 d)$ & $0.0005(7)$ & $0.0077(8)$ & $0.0052(8)$ & $0.0006(6)$ & $0.0005(6)$ & $0.0000(6)$ \\
\hline Al( la) & $0.002(1)$ & $0.005(1)$ & $0.007(1)$ & $0.0013(10)$ & $0.0008(10)$ & $0.000(1)$ \\
\hline $\mathrm{Al}(\mathrm{lb})$ & $0.004(1)$ & $0.007(1)$ & $0.006(1)$ & $0.002(1)$ & $-0.0007(10)$ & $0.002(1)$ \\
\hline $\mathrm{Al}(\mathrm{lc})$ & $0.005(1)$ & $0.006(1)$ & $0.006(1)$ & $0.000(1)$ & $0.001(1)$ & $0.001(1)$ \\
\hline Al(ld) & $0.007(1)$ & $0.005(1)$ & $0.008(1)$ & $-0.003(1)$ & $0.001(1)$ & $-0.001(1)$ \\
\hline$\Lambda 1(2 a)$ & $0.006(1)$ & $0.005(1)$ & $0.006(1)$ & $0.0002(9)$ & $0.0029(8)$ & $0.0010(9)$ \\
\hline $\mathrm{Al}(2 \mathrm{~b})$ & $0.005(1)$ & $0.005(1)$ & $0.006(1)$ & $-0.0017(9)$ & $-0.0018(8)$ & $0.0006(8)$ \\
\hline $\mathrm{Al}(2 \mathrm{c})$ & $0.004(1)$ & $0.005(1)$ & $0.009(1)$ & $0.0000(9)$ & $0.0000(8)$ & $0.0011(9)$ \\
\hline $\mathrm{Al}(2 \mathrm{~d})$ & $0.004(1)$ & $0.005(1)$ & $0.003(1)$ & $-0.0005(8)$ & $0.0011(8)$ & $-0.0016(8)$ \\
\hline $\mathrm{Mg}$ & $0.0030(10)$ & $0.0033(10)$ & $0.023(1)$ & $0.0012(9)$ & $0.0020(9)$ & $-0.0028(10)$ \\
\hline$O(1 a)$ & $0.006(2)$ & $0.006(2)$ & $0.007(2)$ & $-0.001(2)$ & $0.000(2)$ & $0.001(2)$ \\
\hline$O(1 b)$ & $0.009(2)$ & $0.004(2)$ & $0.005(2)$ & $0.000(2)$ & $0.001(2)$ & $0.002(2)$ \\
\hline$O(1 c)$ & $0.006(2)$ & $0.006(2)$ & $0.008(2)$ & $0.003(2)$ & $0.003(2)$ & $0.001(2)$ \\
\hline$O(1 d)$ & $0.007(2)$ & $0.008(2)$ & $0.002(2)$ & $0.000(2)$ & $-0.003(2)$ & $0.001(2)$ \\
\hline$O(2 a)$ & $0.006(2)$ & $0.004(2)$ & $0.009(2)$ & $-0.001(2)$ & $-0.002(2)$ & $0.001(2)$ \\
\hline$O(2 b)$ & $0.005(2)$ & $0.008(2)$ & $0.004(2)$ & $-0.001(2)$ & $0.000(2)$ & $-0.001(2)$ \\
\hline$O(2 c)$ & $0.007(2)$ & $0.006(2)$ & $0.004(2)$ & $0.002(2)$ & $0.000(2)$ & $-0.001(2)$ \\
\hline$O(2 d)$ & $0.005(2)$ & $0.008(2)$ & $0.003(2)$ & $0.001(2)$ & $0.000(2)$ & $0.002(2)$ \\
\hline
\end{tabular}


GROWTH SECTORS IN VESUVIANITE

TABLI: $5 a$. (contd.) Anisotropic displacement parameters of $\{110\}$ sector.

\begin{tabular}{|c|c|c|c|c|c|c|}
\hline Atom & $U_{11}$ & $U_{22}$ & $U_{33}$ & $U_{12}$ & $U_{13}$ & $U_{23}$ \\
\hline$O(3 a)$ & $0.005(2)$ & $0.004(2)$ & $0.010(2)$ & $-0.001(2)$ & $-0.003(2)$ & $-0.002(2)$ \\
\hline$O(3 b)$ & $0.004(2)$ & $0.005(2)$ & $0.004(2)$ & $-0.002(2)$ & $0.000(2)$ & $0.001(2)$ \\
\hline$O(3 c)$ & $0.009(2)$ & $0.004(2)$ & $0.005(2)$ & $0.001(2)$ & $0.001(2)$ & $-0.00 \mathrm{I}(2)$ \\
\hline$O(3 d)$ & $0.006(2)$ & $0.006(2)$ & $0.003(2)$ & $0.002(2)$ & $0.000(2)$ & $-0.001(2)$ \\
\hline$O(4 a)$ & $0.005(2)$ & $0.007(2)$ & $0.007(2)$ & $0.000(2)$ & $0.001(2)$ & $-0.001(2)$ \\
\hline$O(4 b)$ & $0.006(2)$ & $0.007(2)$ & $0.005(2)$ & $0.002(2)$ & $0.000(2)$ & $-0.001(2)$ \\
\hline$O(4 c)$ & $0.006(2)$ & $0.009(2)$ & $0.004(2)$ & $0.000(2)$ & $0.000(2)$ & $-0.003(2)$ \\
\hline$O(4 d)$ & $0.006(2)$ & $0.007(2)$ & $0.005(2)$ & $0.001(2)$ & $0.001(2)$ & $-0.002(2)$ \\
\hline$O(5 a)$ & $0.003(2)$ & $0.013(2)$ & $0.008(2)$ & $0.004(2)$ & $0.001(2)$ & $-0.001(2)$ \\
\hline$O(5 b)$ & $0.005(2)$ & $0.015(2)$ & $0.003(2)$ & $0.007(2)$ & $0.000(2)$ & $0.001(2)$ \\
\hline$O(5 c)$ & $0.011(2)$ & $0.005(2)$ & $0.004(2)$ & $-0.005(2)$ & $0.001(2)$ & $0.000(2)$ \\
\hline$O(5 d)$ & $0.012(2)$ & $0.008(2)$ & $0.006(2)$ & $-0.003(2)$ & $0.002(2)$ & $-0.002(2)$ \\
\hline$O(6 a)$ & $0.018(2)$ & $0.007(2)$ & $0.003(2)$ & $0.002(2)$ & $0.002(2)$ & $0.002(2)$ \\
\hline$O(6 b)$ & $0.019(3)$ & $0.009(2)$ & $0.007(2)$ & $0.002(2)$ & $-0.002(2)$ & $-0.002(2)$ \\
\hline$O(6 \mathrm{c})$ & $0.006(2)$ & $0.019(3)$ & $0.006(2)$ & $-0.001(2)$ & $-0.002(2)$ & $0.002(2)$ \\
\hline$O(6 \mathrm{~d})$ & $0.006(2)$ & $0.018(3)$ & $0.007(2)$ & $-0.003(2)$ & $0.003(2)$ & $-0.005(2)$ \\
\hline$O(7 a)$ & $0.004(2)$ & $0.008(2)$ & $0.011(2)$ & $0.000(2)$ & $0.001(2)$ & $-0.003(2)$ \\
\hline$O(7 b)$ & $0.008(2)$ & $0.009(2)$ & $0.008(2)$ & $0.001(2)$ & $-0.001(2)$ & $0.001(2)$ \\
\hline$O(7 c)$ & $0.011(2)$ & $0.010(2)$ & $0.006(2)$ & $-0.003(2)$ & $0.002(2)$ & $-0.002(2)$ \\
\hline$O(7 d)$ & $0.010(2)$ & $0.006(2)$ & $0.010(2)$ & $-0.002(2)$ & $-0.003(2)$ & $0.000(2)$ \\
\hline$O(8 a)$ & $0.006(2)$ & $0.005(2)$ & $0.008(2)$ & $-0.001(2)$ & $0.002(2)$ & $0.001(2)$ \\
\hline$O(8 b)$ & $0.003(2)$ & $0.005(2)$ & $0.011(2)$ & $0.003(2)$ & $-0.004(2)$ & $-0.003(2)$ \\
\hline$O(8 \mathrm{c})$ & $0.004(2)$ & $0.004(2)$ & $0.009(2)$ & $-0.004(2)$ & $-0.002(2)$ & $0.000(2)$ \\
\hline$O(8 \mathrm{~d})$ & $0.004(2)$ & $0.005(2)$ & $0.011(2)$ & $-0.001(2)$ & $0.000(2)$ & $-0.004(2)$ \\
\hline$O(9 a)$ & $0.012(2)$ & $0.010(2)$ & $0.002(2)$ & $-0.001(2)$ & $0.002(2)$ & $0.001(2)$ \\
\hline$O(9 b)$ & $0.007(2)$ & $0.009(2)$ & $0.006(2)$ & $-0.001(2)$ & $0.000(2)$ & $-0.00 \mid(2)$ \\
\hline$O(10)$ & $0.009(2)$ & $0.014(2)$ & $0.026(3)$ & $-0.001(2)$ & $0.003(2)$ & $0.001(2)$ \\
\hline$O(11 a)$ & $0.006(2)$ & $0.006(2)$ & $0.015(2)$ & $-0.004(2)$ & $0.000(2)$ & $-0.002(2)$ \\
\hline$O(11 b)$ & $0.010(2)$ & $0.008(2)$ & $0.007(2)$ & $-0.001(2)$ & $0.002(2)$ & $0.004(2)$ \\
\hline$O(11 c)$ & $0.007(2)$ & $0.008(2)$ & $0.006(2)$ & $0.002(2)$ & $0.003(2)$ & $0.000(2)$ \\
\hline$O(11 \mathrm{~d})$ & $0.009(2)$ & $0.010(2)$ & $0.008(2)$ & $0.004(2)$ & $-0.002(2)$ & $0.000(2)$ \\
\hline
\end{tabular}

Table 5h. Anisotropic displaccment parameters of $\{111\}$ sector.

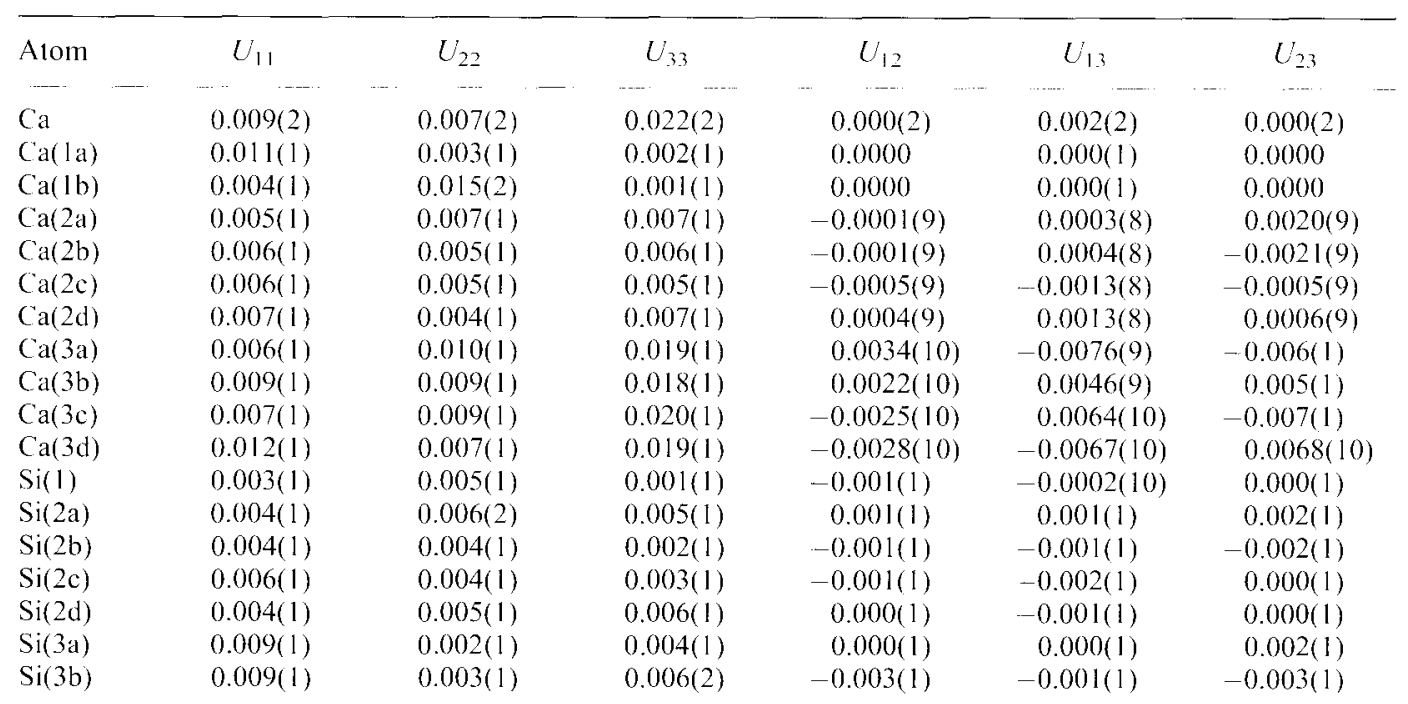


T. TANAKA ETAL.

Table $5 h$. (contd.) Anisotropic displacement parameters of $\{111\}$ sector.

\begin{tabular}{|c|c|c|c|c|c|c|}
\hline Atom & $U_{i 1}$ & $U_{22}$ & $U_{33}$ & $U_{12}$ & $U_{13}$ & $U_{23}$ \\
\hline-- & --- & - - - - & -- & $-\quad--$ & $-\ldots--$ & --- \\
\hline $\operatorname{Si}(3 c)$ & $0.003(1)$ & $0.008(2)$ & $0.006(2)$ & $0.001(1)$ & $-0.004(1)$ & $0.002(1)$ \\
\hline $\operatorname{Si}(3 \mathrm{~d})$ & $0.002(1)$ & $0.010(2)$ & $0.004(1)$ & $0.000(1)$ & $0.003(1)$ & $-0.001(1)$ \\
\hline $\mathrm{Al}(\mathrm{la})$ & $0.003(2)$ & $0.007(2)$ & $0.003(2)$ & $0.001(2)$ & $-0.001(2)$ & $0.000(2)$ \\
\hline $\mathrm{Al}(\mathrm{lb})$ & $0.002(2)$ & $0.004(2)$ & $0.007(2)$ & $0.001(2)$ & $0.002(2)$ & $0.001(2)$ \\
\hline $\operatorname{Al}(1 \mathrm{c})$ & $0.008(2)$ & $0.003(2)$ & $0.011(3)$ & $-0.003(2)$ & $0.002(2)$ & $-0.001(2)$ \\
\hline Al(ld) & $0.004(2)$ & $0.005(2)$ & $0.007(2)$ & $0.000(2)$ & $0.000(2)$ & $0.001(2)$ \\
\hline $\operatorname{Al}(2 a)$ & $0.019(2)$ & $0.010(2)$ & $0.008(2)$ & $-0.001(2)$ & $0.003(2)$ & $-0.002(2)$ \\
\hline $\mathrm{Al}(2 \mathrm{~b})$ & $0.009(2)$ & $0.013(3)$ & $0.014(3)$ & $0.000(2)$ & $-0.001(2)$ & $0.000(2)$ \\
\hline $\operatorname{Al}(2 \mathrm{c})$ & $0.024(3)$ & $0.023(3)$ & $0.013(3)$ & $0.000(3)$ & $0.002(2)$ & $0.001(2)$ \\
\hline $\operatorname{Al}(2 d)$ & $0.012(2)$ & $0.017(3)$ & $0.015(3)$ & $0.002(2)$ & $-0.001(2)$ & $-0.006(2)$ \\
\hline $\mathrm{Mg}$ & $0.008(2)$ & $0.008(2)$ & $0.034(3)$ & $0.002(2)$ & $-0.001(2)$ & $0.007(2)$ \\
\hline$O(I a)$ & $0.014(4)$ & $0.008(4)$ & $0.001(4)$ & $-0.003(3)$ & $-0.002(3)$ & $-0.002(3)$ \\
\hline$O(1 b)$ & $0.006(3)$ & $0.006(4)$ & $0.001(4)$ & $0.000(3)$ & $0.000(3)$ & $0.001(3)$ \\
\hline$O(1 c)$ & $0.006(4)$ & $0.008(4)$ & $0.003(4)$ & $-0.002(3)$ & $0.000(3)$ & $-0.002(3)$ \\
\hline$O(1 d)$ & $0.009(4)$ & $0.013(4)$ & $0.003(4)$ & $0.003(3)$ & $-0.004(3)$ & $0.001(3)$ \\
\hline$O(2 a)$ & $0.009(4)$ & $0.010(4)$ & $0.006(4)$ & $-0.006(3)$ & $-0.006(3)$ & $0.002(3)$ \\
\hline $\mathrm{O}(2 \mathrm{~b})$ & $0.006(3)$ & $0.009(4)$ & $0.007(4)$ & $-0.003(3)$ & $-0.001(3)$ & $-0.001(3)$ \\
\hline$O(2 c)$ & $0.012(4)$ & $0.006(4)$ & $0.005(4)$ & $0.003(3)$ & $-0.001(3)$ & $0.003(3)$ \\
\hline $\mathrm{O}(2 \mathrm{~d})$ & $0.013(4)$ & $0.003(4)$ & $0.005(4)$ & $-0.001(3)$ & $0.005(3)$ & $-0.002(3)$ \\
\hline$O(3 a)$ & $0.006(4)$ & $0.009(4)$ & $0.005(4)$ & $0.000(3)$ & $0.004(3)$ & $-0.001(3)$ \\
\hline$O(3 b)$ & $0.007(4)$ & $0.008(4)$ & $0.005(3)$ & $0.000(3)$ & $-0.003(3)$ & $0.001(3)$ \\
\hline$O(3 c)$ & $0.012(4)$ & $0.011(4)$ & $0.001(4)$ & $-0.002(3)$ & $-0.001(3)$ & $0.002(3)$ \\
\hline $\mathrm{O}(3 \mathrm{~d})$ & $0.009(4)$ & $0.006(4)$ & $0.009(4)$ & $0.005(3)$ & $-0.001(3)$ & $-0.003(3)$ \\
\hline$O(4 a)$ & $0.006(4)$ & $0.001(4)$ & $0.005(4)$ & $-0.001(3)$ & $-0.002(3)$ & $0.001(3)$ \\
\hline$O(4 b)$ & $0.004(4)$ & $0.009(4)$ & $0.010(4)$ & $0.000(3)$ & $0.002(3)$ & $0.003(3)$ \\
\hline $\mathrm{O}(4 \mathrm{c})$ & $0.002(3)$ & $0.014(4)$ & $0.011(4)$ & $-0.001(3)$ & $0.000(3)$ & $0.000(3)$ \\
\hline$O(4 d)$ & $0.006(4)$ & $0.007(4)$ & $0.006(4)$ & $-0.002(3)$ & $-0.005(3)$ & $0.001(3)$ \\
\hline$O(5 a)$ & $0.013(4)$ & $0.007(4)$ & $0.007(4)$ & $0.003(3)$ & $0.001(3)$ & $-0.004(3)$ \\
\hline$O(5 b)$ & $0.012(4)$ & $0.012(4)$ & $0.004(4)$ & $0.006(3)$ & $0.002(3)$ & $0.005(3)$ \\
\hline$O(5 c)$ & $0.010(4)$ & $0.011(4)$ & $0.011(4)$ & $-0.004(3)$ & $0.003(3)$ & $-0.003(3)$ \\
\hline $\mathrm{O}(5 \mathrm{~d})$ & $0.009(4)$ & $0.009(4)$ & $0.008(4)$ & $-0.007(3)$ & $-0.003(3)$ & $0.002(3)$ \\
\hline$O(6 a)$ & $0.018(4)$ & $0.013(4)$ & $0.002(3)$ & $0.005(3)$ & $0.000(3)$ & $0.001(3)$ \\
\hline$O(6 b)$ & $0.020(4)$ & $0.006(4)$ & $0.009(4)$ & $0.002(3)$ & $-0.003(3)$ & $-0.002(3)$ \\
\hline $\mathrm{O}(6 \mathrm{c})$ & $0.012(4)$ & $0.018(5)$ & $0.002(4)$ & $-0.003(4)$ & $0.004(3)$ & $-0.002(3)$ \\
\hline$O(6 d)$ & $0.009(4)$ & $0.015(4)$ & $0.011(4)$ & $-0.003(3)$ & $-0.002(3)$ & $-0.001(4)$ \\
\hline$O(7 a)$ & $0.007(4)$ & $0.017(5)$ & $0.006(4)$ & $-0.002(3)$ & $0.000(3)$ & $0.001(3)$ \\
\hline$O(7 b)$ & $0.011(4)$ & $0.008(4)$ & $0.014(4)$ & $0.001(3)$ & $0.001(3)$ & $0.000(3)$ \\
\hline$O(7 \mathrm{c})$ & $0.012(4)$ & $0.003(4)$ & $0.012(4)$ & $-0.001(3)$ & $0.001(3)$ & $-0.006(3)$ \\
\hline$O(7 d)$ & $0.003(4)$ & $0.011(4)$ & $0.012(4)$ & $0.000(3)$ & $-0.004(3)$ & $0.001(3)$ \\
\hline $\mathrm{O}(8 \mathrm{a})$ & $0.005(4)$ & $0.006(4)$ & $0.011(4)$ & $0.000(3)$ & $-0.002(3)$ & $0.001(3)$ \\
\hline $\mathrm{O}(8 \mathrm{~b})$ & $0.006(4)$ & $0.015(4)$ & $0.009(4)$ & $-0.003(3)$ & $0.002(3)$ & $0.003(3)$ \\
\hline$O(8 c)$ & $0.012(4)$ & $0.002(4)$ & $0.014(4)$ & $0.000(3)$ & $-0.002(3)$ & $-0.004(3)$ \\
\hline $\mathrm{O}(8 \mathrm{~d})$ & $0.005(4)$ & $0.010(4)$ & $0.017(4)$ & $-0.004(3)$ & $-0.006(3)$ & $0.006(3)$ \\
\hline$O(9 a)$ & $0.014(4)$ & $0.014(5)$ & $0.005(4)$ & $-0.005(4)$ & $0.005(3)$ & $-0.005(3)$ \\
\hline$O(9 b)$ & $0.015(4)$ & $0.016(5)$ & $0.002(4)$ & $-0.005(4)$ & $0.000(3)$ & $0.001(3)$ \\
\hline$O(10)$ & $0.017(4)$ & $0.023(5)$ & $0.023(5)$ & $0.007(4)$ & $-0.002(4)$ & $-0.002(5)$ \\
\hline$O(11 a)$ & $0.007(4)$ & $0.011(4)$ & $0.013(4)$ & $-0.002(3)$ & $-0.003(3)$ & $-0.009(3)$ \\
\hline$O(11 b)$ & $0.013(4)$ & $0.007(4)$ & $0.007(4)$ & $-0.002(3)$ & $0.004(3)$ & $0.005(3)$ \\
\hline$O(11 c)$ & $0.014(4)$ & $0.007(4)$ & $0.010(4)$ & $0.000(3)$ & $0.011(3)$ & $-0.001(3)$ \\
\hline$O(11 d)$ & $0.009(4)$ & $0.010(4)$ & $0.008(4)$ & $0.002(3)$ & $-0.004(3)$ & $0.000(3)$ \\
\hline
\end{tabular}




\section{GROWTH SECTORS IN VESUVIANITE}

TABLE $6 a$. Selected interatomic distances $(\AA)$ of the $\{110\}$ sector.

\begin{tabular}{|c|c|c|c|c|c|c|c|}
\hline $\mathrm{Ca}-\mathrm{O}(6 \mathrm{a})$ & $2.313(5)$ & $\mathrm{Ca}(2 \mathrm{c})-\mathrm{O}(5 \mathrm{~d})$ & $2.353(5)$ & $\mathrm{Ca}(3 \mathrm{~d})-\mathrm{O}(10)$ & $2.570(7)$ & $\mathrm{Al}(2 \mathrm{~d})-\mathrm{O}(5 \mathrm{~d})$ & $2.010(5)$ \\
\hline $\mathrm{Ca}-\mathrm{O}(6 \mathrm{~b})$ & $2.318(6)$ & $\mathrm{Ca}(2 \mathrm{c})-\mathrm{O}(6 \mathrm{a})$ & $2,961(5)$ & $\mathrm{Ca}(3 \mathrm{~d})-\mathrm{O}(1 \mid \mathrm{d})$ & $2.499(5)$ & $A l(2 d)-O(1 \mid d)$ & $1.939(5)$ \\
\hline $\mathrm{Ca}-\mathrm{O}(6 \mathrm{c})$ & $2.320(6)$ & $\mathrm{Ca}(2 \mathrm{c})-\mathrm{O}(8 \mathrm{a})$ & $2.324(5)$ & & & & \\
\hline $\mathrm{Ca}-\mathrm{O}(6 \mathrm{~d})$ & $2.313(6)$ & & & $\mathrm{Mg}-\mathrm{O}(6 \mathrm{a})$ & $2.065(5)$ & $\operatorname{Si}(1)-O(1 a)$ & $1.625(5)$ \\
\hline $\mathrm{Ca}-\mathrm{O}(9 \mathrm{a})$ & $2.597(6)$ & $\mathrm{Ca}(2 \mathrm{~d})-\mathrm{O}(\mathrm{lc})$ & $2.486(5)$ & $\mathrm{Mg}-\mathrm{O}(6 \mathrm{~b})$ & $2.074(5)$ & $\operatorname{Si}(1)-O(1 b)$ & $1.623(5)$ \\
\hline $\mathrm{Ca}-\mathrm{O}(9 \mathrm{a})$ & $2.601(5)$ & $\mathrm{Ca}(2 \mathrm{~d})-\mathrm{O}(2 \mathrm{~d})$ & $2.440(5)$ & $\mathrm{Mg}-\mathrm{O}(6 \mathrm{c})$ & $2.070(6)$ & $\operatorname{Si}(1)-O(1 c)$ & $1.621(5)$ \\
\hline $\mathrm{Ca}-\mathrm{O}(9 \mathrm{~b})$ & $2.597(5)$ & $\mathrm{Ca}(2 \mathrm{~d})-\mathrm{O}(3 \mathrm{~b})$ & $2.355(5)$ & $M g-O(6 d)$ & $2.067(5)$ & $\operatorname{Si}(1)-O(1 d)$ & $1.625(5)$ \\
\hline \multirow[t]{2}{*}{$\mathrm{Ca}-\mathrm{O}(9 \mathrm{~b})$} & $2.594(5)$ & $\mathrm{Ca}(2 \mathrm{~d})-\mathrm{O}(4 \mathrm{~d})$ & $2.454(5)$ & $\mathrm{Mg}-\mathrm{O}(10)$ & $2.023(6)$ & & \\
\hline & & $\mathrm{Ca}(2 \mathrm{~d})-\mathrm{O}(5 \mathrm{c})$ & $2.345(5)$ & & & $\operatorname{Si}(2 a)-O(2 c)$ & $1.655(5)$ \\
\hline$C a(1 a)-O(1 a)$ & $2.355(5)$ & $\mathrm{Ca}(2 \mathrm{~d})-\mathrm{O}(5 \mathrm{~d})$ & $2.439(5)$ & $A !(1 a)-O(4 c)$ & $1.943(5)[\times 2]$ & $\operatorname{Si}(2 a)-O(3 c)$ & $1.645(5)$ \\
\hline $\mathrm{Ca}(\mathrm{la})-\mathrm{O}(\mathrm{la})$ & $2.355(5)$ & $\mathrm{Ca}(2 \mathrm{~d})-\mathrm{O}(6 \mathrm{~b})$ & $2.959(5)$ & $A](1 a)-O(8 a)$ & $1.881(4)[\times 2]$ & $\operatorname{Si}(2 a)-O(4 c)$ & $1.666(5)$ \\
\hline $\mathrm{Ca}(1 \mathrm{a})-\mathrm{O}(1 \mathrm{~b})$ & $2.345(5)$ & $\mathrm{Ca}(2 \mathrm{~d})-\mathrm{O}(8 \mathrm{~b})$ & $2.335(5)$ & $A l(1 a)-O(11 a)$ & $1.882(5)[\times 2]$ & $\operatorname{Si}(2 a)-O(7 c)$ & $1.613(5)$ \\
\hline $\mathrm{Ca}(1 \mathrm{a})-\mathrm{O}(\mathrm{lb})$ & $2.345(5)$ & & & & & & \\
\hline $\mathrm{Ca}(1 \mathrm{a})-\mathrm{O}(2 \mathrm{a})$ & $2.527(5)$ & $C a(3 a)-O(3 a)$ & $2.446(5)$ & $\mathrm{Al}(\mathrm{lb})-\mathrm{O}(4 \mathrm{~d})$ & $1.951(5)[\times 2]$ & $\operatorname{Si}(2 b)-O(2 d)$ & $1.647(5)$ \\
\hline $\mathrm{Ca}(1 \mathrm{a})-\mathrm{O}(2 \mathrm{a})$ & $2.527(5)$ & $\mathrm{Ca}(3 \mathrm{a})-\mathrm{O}(6 \mathrm{a})$ & $2.485(5)$ & $\mathrm{Al}(1 \mathrm{~b})-\mathrm{O}(8 \mathrm{~b})$ & $1.875(4)[\times 2]$ & $\operatorname{Si}(2 b)-O(3 d)$ & $1.643(5)$ \\
\hline $\mathrm{Ca}(1 \mathrm{a})-\mathrm{O}(2 \mathrm{~b})$ & $2.518(5)$ & $\mathrm{Ca}(3 \mathrm{a})-\mathrm{O}(6 \mathrm{c})$ & $2.979(5)$ & $A 1(1 b)-O(11 b)$ & $1.875(5)[\times 2]$ & $\operatorname{Si}(2 b)-O(4 d)$ & $1.672(5)$ \\
\hline \multirow[t]{2}{*}{$\mathrm{Ca}(1 \mathrm{a})-\mathrm{O}(2 \mathrm{~b})$} & $2.518(5)$ & $\mathrm{Ca}(3 \mathrm{a})-\mathrm{O}(7 \mathrm{a})$ & $2.575(5)$ & & & $\operatorname{Si}(2 b)-O(7 d)$ & $1.612(5)$ \\
\hline & & $\mathrm{Ca}(3 \mathrm{a})-\mathrm{O}(7 \mathrm{~b})$ & $2.511(5)$ & $\mathrm{Al}(1 \mathrm{c})-\mathrm{O}(4 \mathrm{a})$ & $1.933(5)[\times 2]$ & & \\
\hline $\mathrm{Ca}(1 \mathrm{~b})-\mathrm{O}(1 \mathrm{c})$ & $2.355(4)$ & $\mathrm{Ca}(3 a)-O(7 c)$ & $2.401(5)$ & $A l(1 c)-O(8 c)$ & $1.886(4)[\times 2]$ & $\operatorname{Si}(2 c)-O(2 a)$ & $1.647(5)$ \\
\hline$C a(1 b)-O(1 c)$ & $2.355(4)$ & $\mathrm{Ca}(3 \mathrm{a})-\mathrm{O}(8 \mathrm{a})$ & $2.613(5)$ & $\operatorname{Al}(1 c)-O(11 c)$ & $1.878(4)[\times 2]$ & $\mathrm{Si}(2 \mathrm{c})-\mathrm{O}(3 \mathrm{~b})$ & $1.643(5)$ \\
\hline $\mathrm{Ca}(1 \mathrm{~b})-\mathrm{O}(1 \mathrm{~d})$ & $2.350(4)$ & $\mathrm{Ca}(3 a)-\mathrm{O}(10)$ & $2.560(6)$ & & & $\operatorname{Si}(2 c)-O(4 a)$ & $1.685(5)$ \\
\hline $\mathrm{Ca}(1 \mathrm{~b})-\mathrm{O}(\mathrm{ld})$ & $2.350(4)$ & $\mathrm{Ca}(3 \mathrm{a})-\mathrm{O}(1 \mathrm{la})$ & $2.503(5)$ & $A l(1 d)-O(4 b)$ & $1.932(5)[\times 2]$ & $\mathrm{Si}(2 \mathrm{c})-\mathrm{O}(7 \mathrm{a})$ & $1.620(5)$ \\
\hline $\mathrm{Ca}(1 \mathrm{~b})-\mathrm{O}(2 \mathrm{c})$ & $2.515(5)$ & & & $\mathrm{Al}(\mathrm{Id})-\mathrm{O}(8 \mathrm{~d})$ & $1.879(4)[\times 2]$ & & \\
\hline $\mathrm{Ca}(1 \mathrm{~b})-\mathrm{O}(2 \mathrm{c})$ & $2.516(5)$ & $\mathrm{Ca}(3 \mathrm{~b})-\mathrm{O}(3 \mathrm{~b})$ & $2.442(5)$ & $\mathrm{Al}(1 \mathrm{~d})-\mathrm{O}(1 \mathrm{~d})$ & $1.877(5)[\times 2]$ & $\operatorname{Si}(2 d)-O(2 b)$ & $1.642(5)$ \\
\hline $\mathrm{Ca}(1 \mathrm{~b})-\mathrm{O}(2 \mathrm{~d})$ & $2.525(5)$ & $\mathrm{Ca}(3 \mathrm{~b})-\mathrm{O}(6 \mathrm{~b})$ & $2.482(5)$ & & & $\operatorname{Si}(2 d)-O(3 a)$ & $1.651(5)$ \\
\hline \multirow[t]{2}{*}{$\mathrm{Ca}(1 \mathrm{~b})-\mathrm{O}(2 \mathrm{~d})$} & $2.525(5)$ & $\mathrm{Ca}(3 \mathrm{~b})-\mathrm{O}(6 \mathrm{~d})$ & $2.988(5)$ & $\mathrm{Al}(2 \mathrm{a})-\mathrm{O}(\mathrm{la})$ & $1.943(5)$ & $\mathrm{Si}(2 \mathrm{~d})-\mathrm{O}(4 \mathrm{~b})$ & $1.685(5)$ \\
\hline & & $\mathrm{Ca}(3 \mathrm{~b})-\mathrm{O}(7 \mathrm{a})$ & $2.505(5)$ & $\operatorname{Al}(2 a)-O(2 a)$ & $1.907(5)$ & $\operatorname{Si}(2 d)-O(7 b)$ & $1.616(5)$ \\
\hline$C a(2 a)-O(1 a)$ & $2.485(5)$ & $\mathrm{Ca}(3 \mathrm{~b})-\mathrm{O}(7 \mathrm{~b})$ & $2.572(5)$ & $\mathrm{Al}(2 \mathrm{a})-\mathrm{O}(3 \mathrm{a})$ & $1.979(5)$ & & \\
\hline $\mathrm{Ca}(2 \mathrm{a})-\mathrm{O}(2 \mathrm{a})$ & $2.441(5)$ & $\mathrm{Ca}(3 \mathrm{~b})-\mathrm{O}(7 \mathrm{~d})$ & $2.400(5)$ & $\operatorname{Al}(2 a)-O(4 c)$ & $2.093(5)$ & $\mathrm{Si}(3 a)-O(5 c)$ & $1.635(5)$ \\
\hline $\mathrm{Ca}(2 \mathrm{a})-\mathrm{O}(3 \mathrm{c})$ & $2.365(5)$ & $\mathrm{Ca}(3 \mathrm{~b})-\mathrm{O}(8 \mathrm{~b})$ & $2.614(5)$ & $\mathrm{Al}(2 \mathrm{a})-\mathrm{O}(5 \mathrm{a})$ & $2.017(5)$ & $\mathrm{Si}(3 \mathrm{a})-\mathrm{O}(6 \mathrm{~b})$ & $1.619(5)$ \\
\hline $\mathrm{Ca}(2 \mathrm{a})-\mathrm{O}(4 \mathrm{a})$ & $2.455(5)$ & $\mathrm{Ca}(3 \mathrm{~b})-\mathrm{O}(10)$ & $2.570(5)$ & $\mathrm{Al}(2 \mathrm{a})-\mathrm{O}(1 \mathrm{la})$ & $1.930(5)$ & $\mathrm{Si}(3 \mathrm{a})-\mathrm{O}(8 \mathrm{c})$ & $1.622(5)$ \\
\hline $\mathrm{Ca}(2 \mathrm{a})-\mathrm{O}(5 \mathrm{a})$ & $2.432(5)$ & $\mathrm{Ca}(3 b)-O(11 b)$ & $2.497(5)$ & & & $\operatorname{Si}(3 a)-O(9 a)$ & $1.656(5)$ \\
\hline $\mathrm{Ca}(2 \mathrm{a})-\mathrm{O}(5 \mathrm{a})$ & $2.349(5)$ & & & $A l(2 b)-O(1 b)$ & $1.957(5)$ & & \\
\hline $\mathrm{Ca}(2 \mathrm{a})-\mathrm{O}(6 \mathrm{c})$ & $2.965(5)$ & $\mathrm{Ca}(3 \mathrm{c})-\mathrm{O}(3 \mathrm{c})$ & $2.443(5)$ & $\mathrm{Al}(2 \mathrm{~b})-\mathrm{O}(2 \mathrm{~b})$ & $1.922(5)$ & $\mathrm{Si}(3 \mathrm{~b})-\mathrm{O}(5 \mathrm{~d})$ & $1.629(5)$ \\
\hline \multirow[t]{2}{*}{$\mathrm{Ca}(2 \mathrm{a})-\mathrm{O}(8 \mathrm{c})$} & $2.333(4)$ & $\mathrm{Ca}(3 \mathrm{c})-\mathrm{O}(6 \mathrm{~b})$ & $2.988(5)$ & $\mathrm{Al}(2 \mathrm{~b})-\mathrm{O}(3 \mathrm{~b})$ & $1.983(5)$ & $\mathrm{Si}(3 \mathrm{~b})-\mathrm{O}(6 \mathrm{a})$ & $1.613(5)$ \\
\hline & & $\mathrm{Ca}(3 \mathrm{c})-\mathrm{O}(6 \mathrm{c})$ & $2.477(5)$ & $\mathrm{Al}(2 \mathrm{~b})-\mathrm{O}(4 \mathrm{~d})$ & $2.092(5)$ & $\mathrm{Si}(3 \mathrm{~b})-\mathrm{O}(8 \mathrm{~d})$ & $1.629(5)$ \\
\hline $\mathrm{Ca}(2 \mathrm{~b})-\mathrm{O}(1 \mathrm{~b})$ & $2.479(5)$ & $\mathrm{Ca}(3 c)-O(7 a)$ & $2.401(5)$ & $A l(2 b)-O(5 b)$ & $2.021(5)$ & $\operatorname{Si}(3 b)-O(9 b)$ & $1.662(5)$ \\
\hline $\mathrm{Ca}(2 \mathrm{~b})-\mathrm{O}(2 \mathrm{~b})$ & $2.436(5)$ & $\mathrm{Ca}(3 \mathrm{c})-\mathrm{O}(7 \mathrm{c})$ & $2.504(5)$ & $\mathrm{Al}(2 \mathrm{~b})-\mathrm{O}(11 \mathrm{~b})$ & $1.951(5)$ & & \\
\hline $\mathrm{Ca}(2 \mathrm{~b})-\mathrm{O}(3 \mathrm{~d})$ & $2.363(5)$ & $\mathrm{Ca}(3 \mathrm{c})-\mathrm{O}(7 \mathrm{c})$ & $2.580(5)$ & & & $\operatorname{Si}(3 c)-O(5 a)$ & $1.632(5)$ \\
\hline $\mathrm{Ca}(2 \mathrm{~b})-\mathrm{O}(4 \mathrm{~b})$ & $2.471(5)$ & $\mathrm{Ca}(3 \mathrm{c})-\mathrm{O}(8 \mathrm{c})$ & $2.620(5)$ & $\mathrm{Al}(2 \mathrm{c})-\mathrm{O}(\mathrm{Ic})$ & $1.949(5)$ & $\operatorname{Si}(3 c)-O(6 c)$ & $1.615(5)$ \\
\hline $\mathrm{Ca}(2 \mathrm{~b})-\mathrm{O}(5 \mathrm{~b})$ & $2.426(5)$ & $\mathrm{Ca}(3 \mathrm{c})-\mathrm{O}(10)$ & $2.562(7)$ & $\mathrm{Al}(2 \mathrm{c})-\mathrm{O}(2 \mathrm{c})$ & $1.916(5)$ & $\mathrm{Si}(3 \mathrm{c})-\mathrm{O}(8 \mathrm{a})$ & $1.636(5)$ \\
\hline $\mathrm{Ca}(2 \mathrm{~b})-\mathrm{O}(5 \mathrm{~b})$ & $2.341(5)$ & $\mathrm{Ca}(3 \mathrm{c})-\mathrm{O}(1 \mathrm{lc})$ & $2.499(5)$ & $A l(2 c)-O(3 c)$ & $1.975(5)$ & $\operatorname{Si}(3 c)-O(9 a)$ & $1.661(5)$ \\
\hline $\mathrm{Ca}(2 \mathrm{~b})-\mathrm{O}(6 \mathrm{~d})$ & $2.967(5)$ & & & $\operatorname{Al}(2 c)-O(4 a)$ & $2.082(5)$ & & \\
\hline \multirow[t]{2}{*}{$\mathrm{Ca}(2 \mathrm{~b})-\mathrm{O}(8 \mathrm{~d})$} & $2.335(5)$ & $\mathrm{Ca}(3 \mathrm{~d})-\mathrm{O}(3 \mathrm{~d})$ & $2.447(5)$ & $\operatorname{Al}(2 \mathrm{c})-\mathrm{O}(5 \mathrm{c})$ & $2.022(5)$ & $\mathrm{Si}(3 \mathrm{~d})-\mathrm{O}(5 \mathrm{~b})$ & $1.637(5)$ \\
\hline & & $\mathrm{Ca}(3 \mathrm{~d})-\mathrm{O}(6 \mathrm{a})$ & $2.983(5)$ & $A l(2 c)-O(1 \mid c)$ & $1.939(5)$ & $\mathrm{Si}(3 \mathrm{~d})-\mathrm{O}(6 \mathrm{~d})$ & $1.617(5)$ \\
\hline $\mathrm{Ca}(2 \mathrm{c})-\mathrm{O}(\mathrm{ld})$ & $2.486(5)$ & $\mathrm{Ca}(3 \mathrm{~d})-\mathrm{O}(6 \mathrm{~d})$ & $2.483(5)$ & & & $\mathrm{Si}(3 \mathrm{~d})-\mathrm{O}(8 \mathrm{~b})$ & $1.632(5)$ \\
\hline $\mathrm{Ca}(2 \mathrm{c})-\mathrm{O}(2 \mathrm{c})$ & $2.441(5)$ & $\mathrm{Ca}(3 \mathrm{~d})-\mathrm{O}(7 \mathrm{~b})$ & $2.396(5)$ & $\mathrm{Al}(2 \mathrm{~d})-\mathrm{O}(1 \mathrm{~d})$ & $1.946(5)$ & $\mathrm{Si}(3 \mathrm{~d})-\mathrm{O}(9 \mathrm{~b})$ & $1.667(5)$ \\
\hline $\mathrm{Ca}(2 \mathrm{c})-\mathrm{O}(3 \mathrm{a})$ & $2.360(5)$ & $\mathrm{Ca}(3 \mathrm{~d})-\mathrm{O}(7 \mathrm{~d})$ & $2.511(5)$ & $\operatorname{Al}(2 \mathrm{~d})-\mathrm{O}(2 \mathrm{~d})$ & $1.917(5)$ & & \\
\hline $\mathrm{Ca}(2 \mathrm{c})-\mathrm{O}(4 \mathrm{c})$ & $2.466(5)$ & $\mathrm{Ca}(3 \mathrm{~d})-\mathrm{O}(7 \mathrm{~d})$ & $2.577(5)$ & $\mathrm{Al}(2 \mathrm{~d})-\mathrm{O}(3 \mathrm{~d})$ & $1.977(5)$ & & \\
\hline $\mathrm{Ca}(2 \mathrm{c})-\mathrm{O}(5 \mathrm{c})$ & $2.426(5)$ & $\mathrm{Ca}(3 \mathrm{~d})-\mathrm{O}(8 \mathrm{~d})$ & $2.616(5)$ & $A l(2 d)-O(4 b)$ & $2.081(5)$ & & \\
\hline
\end{tabular}

vesuvianite. However, the $\mathrm{OH}^{-}$dipole is randomly oriented in the present vesuvianite. Therefore, it is suggested that the low symmetry of the specimen is not due to preferred orientation of $\mathrm{OH}^{-}$dipoles.
Akizuki (1981) proposed a formation mechanism for cation ordering in zeolites and some other minerals. Since the atomic ordering occurs by a charge balance of atoms along growth steps, if the growth steps are normal to the 
T.TANAKA ETAL.

Table $6 h$. Selected interatomic distances $(\AA)$ of the $\{111\}$ sector.

\begin{tabular}{|c|c|c|c|c|c|c|c|}
\hline$-\ldots$ & - - - & - & --- & - & - & - & $\bar{C}$ \\
\hline $\mathrm{Ca}-\mathrm{O}(6 \mathrm{a})$ & $2.294(9)$ & $\mathrm{Ca}(2 \mathrm{c})-\mathrm{O}(5 \mathrm{c})$ & $2.427(8)$ & $\mathrm{Ca}(3 \mathrm{~d})-\mathrm{O}(7 \mathrm{~d})$ & $2.608(8)$ & $\Lambda \mathrm{l}(2 \mathrm{~d})-\mathrm{O}(3 \mathrm{~d})$ & $1.990(8)$ \\
\hline $\mathrm{Ca}-\mathrm{O}(6 \mathrm{~b})$ & $2.292(9)$ & $\mathrm{Ca}(2 \mathrm{c})-\mathrm{O}(5 \mathrm{~d})$ & $2.341(8)$ & $\mathrm{Ca}(3 \mathrm{~d})-\mathrm{O}(8 \mathrm{~d})$ & $2.606(8)$ & $A l(2 d)-O(4 b)$ & $2.089(8)$ \\
\hline $\mathrm{Ca}-\mathrm{O}(6 \mathrm{c})$ & $2.28(1)$ & $\mathrm{Ca}(2 \mathrm{c})-\mathrm{O}(6 \mathrm{a})$ & $2.979(8)$ & $\mathrm{Ca}(3 \mathrm{~d})-\mathrm{O}(10)$ & $2.58(1)$ & $A \mid(2 d)-O(5 d)$ & $2.026(8)$ \\
\hline $\mathrm{Ca}-\mathrm{O}(\mathrm{bd})$ & $2.30(1)$ & $C a(2 c)-O(8 a)$ & $2.324(8)$ & $\mathrm{Ca}(3 \mathrm{~d})-\mathrm{O}(1 / \mathrm{d})$ & $2.503(8)$ & $A \mid(2 d)-O(1 \mid d)$ & $1.933(9)$ \\
\hline $\mathrm{Ca}-\mathrm{O}(9 \mathrm{a})$ & $2.608(9)$ & & & & & & \\
\hline $\mathrm{Ca}-\mathrm{O}(9 \mathrm{a})$ & $2.602(9)$ & $\mathrm{Ca}(2 \mathrm{~d})-\mathrm{O}(\mathrm{lc})$ & $2.472(8)$ & $\operatorname{Mg}-O(6 a)$ & $2.062(8)$ & $\operatorname{Si}(1)-O(1 a)$ & $1.634(8)$ \\
\hline $\mathrm{Ca}-\mathrm{O}(9 \mathrm{~b})$ & $2.618(9)$ & $C a(2 d)-O(2 d)$ & $2.437(8)$ & $\mathrm{Mg}-\mathrm{O}(6 \mathrm{~b})$ & $2.064(9)$ & $\operatorname{Si}(1)-O(1 b)$ & $1.634(8)$ \\
\hline $\mathrm{Ca}-\mathrm{O}(9 \mathrm{~b})$ & $2.621(9)$ & $(\mathrm{a}(2 \mathrm{~d})-O(3 \mathrm{~b})$ & $2.372(7)$ & $\mathrm{Mg}-O(6 \mathrm{c})$ & $2.053(9)$ & $\operatorname{Si}(1)-O(1 c)$ & $1.637(8)$ \\
\hline & & $\mathrm{Ca}(2 \mathrm{~d})-\mathrm{O}(4 \mathrm{~d})$ & $2.463(8)$ & $\mathrm{Mg}-\mathrm{O}(\mathrm{Gd})$ & $2.071(9)$ & $\operatorname{Si}(1)-O(1 d)$ & $1.624(8)$ \\
\hline $\mathrm{Ca}(1 \mathrm{a})-\mathrm{O}(1 \mathrm{a})$ & $2.335(8)$ & $\mathrm{Ca}(2 \mathrm{~d})-\mathrm{O}(5 \mathrm{c})$ & $2.354(8)$ & $\mathrm{Mg}-\mathrm{O}(10)$ & $2.071(9)$ & & \\
\hline $\mathrm{Ca}(\mathrm{la})-\mathrm{O}(\mathrm{la})$ & $2.334(8)$ & $C a(2 d)-O(5 d)$ & $2.437(8)$ & & & $\operatorname{Si}(2 a)-O(2 c)$ & $1.651(8)$ \\
\hline $\mathrm{Ca}(1 \mathrm{a})-\mathrm{O}(\mathrm{lb})$ & $2.350(7)$ & $\mathrm{Ca}(2 \mathrm{~d})-\mathrm{O}(6 \mathrm{~b})$ & $2.961(8)$ & $\mathrm{Al}(1 \mathrm{a})-\mathrm{O}(4 \mathrm{c})$ & $1.943(7)[\times 2]$ & $\operatorname{Si}(2 a)-O(3 c)$ & $1.639(8)$ \\
\hline $\mathrm{Ca}(\mathrm{Ia})-\mathrm{O}(\mathrm{lb})$ & $2.350(7)$ & $\mathrm{Ca}(2 \mathrm{~d})-\mathrm{O}(8 \mathrm{~b})$ & $2.333(8)$ & $A l(1 a)-O(8 a)$ & $1.894(8)[\times 2]$ & $\operatorname{Si}(2 a)-O(4 c)$ & $1.671(8)$ \\
\hline $\mathrm{Ca}(1 \mathrm{a})-\mathrm{O}(2 \mathrm{a})$ & $2.510(8)$ & & & $A I(l a)-O(1 \mid a)$ & $1.867(8)[\times 2]$ & $\operatorname{Si}(2 a)-O(7 c)$ & $1.609(8)$ \\
\hline $\mathrm{Ca}(1 \mathrm{a})-\mathrm{O}(2 \mathrm{a})$ & $2.511(8)$ & $\mathrm{Ca}(3 \mathrm{a})-\mathrm{O}(3 \mathrm{a})$ & $2.422(8)$ & & & & \\
\hline $\mathrm{Ca}(\mathrm{la})-\mathrm{O}(2 \mathrm{~b})$ & $2.530(8)$ & Ca(3a)-O(6a) & $2.504(8)$ & $\mathrm{Al}(1 \mathrm{lb})-\mathrm{O}(4 \mathrm{~d})$ & $1.943(7)[\times 2]$ & $\operatorname{Si}(2 b)-O(2 d)$ & $1.643(8)$ \\
\hline$(a(1 a)-O(2 b)$ & $2.529(8)$ & $C a(3 a)-O(6 c)$ & $3.000(8)$ & $A l(1 b)-O(8 b)$ & $1.885(8)\lceil\times 2\rceil$ & $\operatorname{Si}(2 b)-O(3 d)$ & $1.635(8)$ \\
\hline & & $C a(3 a)-O(7 a)$ & $2.610(8)$ & $\mathrm{Al}(1 \mathrm{~b})-\mathrm{O}(11 \mathrm{~b})$ & $1.864(7)[\times 2]$ & $\mathrm{Si}(2 \mathrm{~b})-\mathrm{O}(4 \mathrm{~d})$ & $1.668(8)$ \\
\hline $\mathrm{Ca}(1 \mathrm{~b})-\mathrm{O}(\mathrm{lc})$ & $2.343(7)$ & $C a(3 a)-O(7 b)$ & $2.506(8)$ & $\operatorname{Al}(1 c)-O(4 a)$ & $1.947(7)[\times 2]$ & $\mathrm{Si}(2 \mathrm{~b})-\mathrm{O}(7 \mathrm{~d})$ & $1.617(8)$ \\
\hline $\mathrm{Ca}(\mathrm{lb})-\mathrm{O}(\mathrm{lc})$ & $2.343(7)$ & $\operatorname{Ca}(3 a)-O(7 c)$ & $2.382(8)$ & $A l(1 c)-O(8 c)$ & $1.893(8)[\times 2]$ & & \\
\hline$C a(1 b)-O(1 d)$ & $2.344(7)$ & $C a(3 a)-O(8 a)$ & $2.610(8)$ & $\mathrm{Al}(1 \mathrm{c})-\mathrm{O}(11 \mathrm{c})$ & $1.844(7)[\times 2]$ & $\operatorname{Si}(2 c)-O(2 a)$ & $1.643(8)$ \\
\hline $\mathrm{Ca}(\mathrm{lb})-\mathrm{O}(\mathrm{ld})$ & $2.344(7)$ & $\mathrm{Ca}(3 \mathrm{a})-\mathrm{O}(10)$ & $2.571(9)$ & & & $\operatorname{Si}(2 c)-O(3 b)$ & $1.644(8)$ \\
\hline $\mathrm{Ca}(1 \mathrm{~b})-\mathrm{O}(2 \mathrm{c})$ & $2.514(9)$ & $C a(3 a)-O(11 a)$ & $2.497(8)$ & $A 1(I d)-O(4 b)$ & $1.951(8)[\times 2]$ & $\operatorname{Si}(2 c)-O(4 a)$ & $1.667(8)$ \\
\hline$(\mathrm{a}(\mathrm{lb})-\mathrm{O}(2 \mathrm{c})$ & $2.513(9)$ & & & $\mathrm{Al}(\mathrm{ld})-\mathrm{O}(8 \mathrm{~d})$ & $1.880(8)[\times 2]$ & $\operatorname{Si}(2 c)-O(7 a)$ & $1.605(8)$ \\
\hline$(a(1 b)-O(2 d)$ & $2.519(8)$ & $\mathrm{Ca}(3 b)-O(3 b)$ & $2.442(8)$ & $A I(I d)-O(1 / d)$ & $1.859(7)\lceil\times 2]$ & & \\
\hline $\mathrm{Ca}(\mathrm{lb})-\mathrm{O}(2 \mathrm{~d})$ & $2.519(8)$ & $\mathrm{Ca}(3 b)-\mathrm{O}(6 \mathrm{~b})$ & $2.491(8)$ & & & 2b) & $1.660(8)$ \\
\hline & & $C a(3 b)-O(6 d)$ & $2.988(8)$ & $\mathrm{Al}(2 \mathrm{a})-\mathrm{O}(\mathrm{I} a)$ & 1.95 & $\operatorname{Si}(2 d)-O(3 a)$ & $1.634(8)$ \\
\hline $\mathrm{Ca}(2 \mathrm{a})$ & $2.464(8)$ & $(a(3 b)-O(7 a)$ & $2.491(9)$ & $A](2 a)-O(2 a)$ & (8) & $\operatorname{Si}(2 d)-O(4 b)$ & $1.665(8)$ \\
\hline $\mathrm{Ca}(2 \mathrm{a})-\mathrm{O}(2 \mathrm{a})$ & $2.437(8)$ & $\mathrm{Ca}(3 \mathrm{~b})-\mathrm{O}(7 \mathrm{~b})$ & $2.599(8)$ & $\mathrm{Al}(2 \mathrm{a})-\mathrm{O}(3 \mathrm{a})$ & $1.992(8)$ & $\operatorname{Si}(2 d)-O(7 b)$ & $1.603(8)$ \\
\hline $\mathrm{Ca}(2 \mathrm{a})-\mathrm{O}(3 \mathrm{c})$ & $2.377(8)$ & $(a(3 b)-O(7 d)$ & $2.373(8)$ & $\operatorname{Al}(2 a)-O(4 c)$ & $2.096(8)$ & & \\
\hline $\mathrm{Ca}(2 \mathrm{a})-\mathrm{O}(4 \mathrm{a})$ & $2.455(7)$ & $\mathrm{Ca}(3 b)-O(8 b)$ & $2.624(8)$ & $A \mathrm{l}(2 \mathrm{a})-O(5 \mathrm{a})$ & $2.025(9)$ & $O(5 c)$ & $1.631(8)$ \\
\hline $\mathrm{Ca}(2 \mathrm{a})-\mathrm{O}(5 \mathrm{a})$ & $2.423(8)$ & $\mathrm{Ca}(3 \mathrm{~b})-\mathrm{O}(10)$ & $2.57(1)$ & $\operatorname{Al}(2 a)-O(11 a)$ & $1.936(8)$ & $\operatorname{Si}(3 a)-O(6 b)$ & $1.616(8)$ \\
\hline $\mathrm{Ca}(2 \mathrm{a})-\mathrm{O}(5 \mathrm{a})$ & $2.341(8)$ & $(a(3 b)-O(11 b)$ & $2.501(8)$ & & & $\operatorname{Si}(3 a)-O(8 c)$ & $1.630(8)$ \\
\hline $\mathrm{Ca}(2 \mathrm{a})-\mathrm{O}(6 \mathrm{c})$ & $2.963(9)$ & & & $A l(2 b)-O(1 b)$ & $1.952(8)$ & $\operatorname{Si}(3 a)-0(9 a)$ & $1.656(9)$ \\
\hline $\mathrm{Ca}(2 \mathrm{a})-\mathrm{O}(8 \mathrm{c})$ & $2.317(8)$ & $\mathrm{Ca}(3 \mathrm{c})-\mathrm{O}(3 \mathrm{c})$ & $2.424(8)$ & $\mathrm{AI}(2 \mathrm{~b})-\mathrm{O}(2 \mathrm{~b})$ & $1.899(8)$ & & \\
\hline & & $\mathrm{Ca}(3 \mathrm{c})-\mathrm{O}(6 \mathrm{~b})$ & $2.987(8)$ & $\Lambda \mathrm{l}(2 \mathrm{~b})-\mathrm{O}(3 \mathrm{~b})$ & & b) $-O(5 d)$ & $1.625(8)$ \\
\hline $\mathrm{Ca}(2 \mathrm{~b})-\mathrm{O}(1 \mathrm{~b})$ & $2.466(8)$ & $\mathrm{Ca}(3 \mathrm{c})-0(6 \mathrm{c})$ & $2.480(8)$ & $\operatorname{Al}(2 b)-O(4 d)$ & $2.094(8)$ & $\operatorname{Si}(3 b)-O(6 a)$ & $1.607(8)$ \\
\hline $\mathrm{Ca}(2 \mathrm{~b})-\mathrm{O}(2 \mathrm{~b})$ & $2.441(8)$ & $C a(3 c)-O(7 a)$ & $2.394(8)$ & $\mathrm{Al}(2 \mathrm{~b})-\mathrm{O}(5 \mathrm{~b})$ & $2.013(9)$ & $\operatorname{Si}(3 b)-O(8 d)$ & $1.619(8)$ \\
\hline$(a(2 b)-O(3 d)$ & $2.364(8)$ & $(a(3 c)-O(7 c)$ & $2.494(8)$ & $A](2 b)-O(11 b)$ & $1.935(8)$ & $\mathrm{Si}(3 b)-\mathrm{O}(9 b)$ & $1.653(8)$ \\
\hline $\mathrm{Ca}(2 \mathrm{~b})-\mathrm{O}(4 \mathrm{~b})$ & $2.449(8)$ & $\mathrm{Ca}(3 \mathrm{c})-\mathrm{O}(7 \mathrm{c})$ & $2.606(8)$ & & & & \\
\hline $\mathrm{Ca}(2 b)-O(5 b)$ & $2.433(8)$ & $(a(3 c)-O(8 c)$ & $2.612(8)$ & $A \mid(2 c)-O(1 c)$ & $(8)$ & $-0(5 a)$ & $1.632(8)$ \\
\hline $\mathrm{Ca}(2 \mathrm{~b})-\mathrm{O}(5 \mathrm{~b})$ & $2.355(8)$ & $\mathrm{Ca}(3 \mathrm{c})-\mathrm{O}(10)$ & $2.57(1)$ & $\mathrm{Al}(2 \mathrm{c})-\mathrm{O}(2 \mathrm{c})$ & $1.913(8)$ & $\operatorname{Si}(3 c)-O(6 c)$ & $1.627(8)$ \\
\hline$C a(2 b)-O(6 d)$ & $2.967(9)$ & $\mathrm{Ca}(3 \mathrm{c})-\mathrm{O}(1 \mid \mathrm{c})$ & $2.509(8)$ & $\mathrm{Al}(2 \mathrm{c})-\mathrm{O}(3 \mathrm{c})$ & $1.977(8)$ & $\mathrm{Si}(3 \mathrm{c})-\mathrm{O}(8 \mathrm{a})$ & $1.620(8)$ \\
\hline $\mathrm{Ca}(2 \mathrm{~b})-\mathrm{O}(8 \mathrm{~d})$ & $2.338(7)$ & & & $\operatorname{Al}(2 c)-()(4 a)$ & $2.092(8)$ & $\operatorname{Si}(3 c)-O(9 a)$ & $1.662(9)$ \\
\hline & & $O(3 d)$ & $2.439(8)$ & $\mathrm{Al}(2 \mathrm{c})-\mathrm{O}(5 \mathrm{c})$ & & & \\
\hline $\mathrm{Ca}(2 \mathrm{c})-\mathrm{O}(\mathrm{ld})$ & $2.475(8)$ & $\mathrm{Ca}(3 \mathrm{~d})-\mathrm{O}(6 \mathrm{a})$ & $2.980(8)$ & $\operatorname{Al}(2 c)-O(1 \mid c)$ & $1.929(9)$ & $\mathrm{Si}(3 \mathrm{~d})-\mathrm{O}(5 \mathrm{~b})$ & $1.636(8)$ \\
\hline $\mathrm{Ca}(2 \mathrm{c})-\mathrm{O}(2 \mathrm{c})$ & $2.445(8)$ & $C a(3 d)-O(6 d)$ & $2.499(9)$ & & & $\operatorname{Si}(3 d)-O(6 d)$ & $1.601(8)$ \\
\hline $\mathrm{Ca}(2 \mathrm{c})-\mathrm{O}(3 \mathrm{a})$ & $2.370(8)$ & $(a(3 d)-O(7 b)$ & $2.385(8)$ & $A l(2 d)-O(1 d)$ & $1.962(8)$ & $\mathrm{Si}(3 \mathrm{~d})-\mathrm{O}(8 \mathrm{~b})$ & $1.610(8)$ \\
\hline $\mathrm{Ca}(2 \mathrm{c})-\mathrm{O}(4 \mathrm{c})$ & $2.452(8)$ & $\mathrm{Ca}(3 \mathrm{~d})-\mathrm{O}(7 \mathrm{~d})$ & $2.503(8)$ & $\operatorname{Al}(2 d)-O(2 d)$ & $1.928(8)$ & $\operatorname{Si}(3 d)-O(9 b)$ & $1.670(9)$ \\
\hline
\end{tabular}

symmetrical mirror plane, the mirror plane will be maintained during growth. If the steps are inclined to the mirror plane, the structure will not have the symmetry of the morphological symmetry plane.
The crystal structure of vesuvianite is similar to that of grandite garnet, and Akizuki (1984) suggested that the low-symmetry sector of grandite garnet is produced by same mechanism 
TABl.l: 7. Al/Fe occupancies of the $\{110\}$ and $\{111\}$ sectors in vesuvianite.

\begin{tabular}{|c|c|c|c|}
\hline \multicolumn{4}{|c|}{110} sector \\
\hline $\mathrm{Al}(\mathrm{la})$ & $\mathrm{Al}_{0.483(3)} \mathrm{Fe}_{0.017}$ & $\operatorname{Al}(2 \mathrm{a})$ & $\mathrm{Al}_{(0.780(5)} \mathrm{Fe}_{(0.220)}$ \\
\hline $\mathrm{Al}(\mathrm{Ib})$ & $\mathrm{Al}_{0.479(3)} \mathrm{Fe}_{6.0(121}$ & $\mathrm{Al}(2 \mathrm{~b})$ & $\mathrm{Al}_{0.80 \varphi_{(5,5}} \mathrm{Fe}_{0.191}$ \\
\hline $\mathrm{Al}(1 \mathrm{c})$ & $\mathrm{Al}_{0.474(3)} \mathrm{Fe}_{0.026}$ & $\mathrm{Al}(2 \mathrm{c})$ & $\mathrm{Al}_{(0.798(5)} \mathrm{Fe}_{0.202}$ \\
\hline $\mathrm{Al}(1 \mathrm{~d})$ & $\mathrm{Al}_{0.473(3)} \mathrm{Fe}_{0.027}$ & $\operatorname{Al}(2 \mathrm{~d})$ & $\mathrm{Al}_{0.820(5)} \mathrm{Fe}_{0.180}$ \\
\hline \multicolumn{4}{|c|}{ \{111\} sector } \\
\hline $\operatorname{Al}(\mathrm{Ia})$ & $\mathrm{Al}_{0.484(5)} \mathrm{Fe}_{0.016}$ & $\mathrm{Al}(2 \mathrm{a})$ & $\mathrm{Al}_{0.801,7,} \mathrm{Fe}_{0.199}$ \\
\hline $\mathrm{Al}(\mathrm{Ib})$ & $A \mathrm{l}_{0.487(5)} \mathrm{Fe}_{0.013}$ & $\mathrm{Al}(2 \mathrm{~b})$ & $\mathrm{Al}_{0.73 \times(7)} \mathrm{Fe}_{0.262}$ \\
\hline $\mathrm{Al}(1 \mathrm{c})$ & $\mathrm{Al}_{0.478(5)} \mathrm{Fe}_{0.022}$ & $\operatorname{Al}(2 \mathrm{c})$ & $\mathrm{Al}_{0.706(7)} \mathrm{Fc}_{0.294}$ \\
\hline $\mathrm{Al}(\mathrm{Id})$ & $\mathrm{Al}_{(0.486(5)} \mathrm{Fe}_{(0.014}$ & $\operatorname{Al}(2 \mathrm{~d})$ & $\mathrm{Al}_{0.743(7,}, \mathrm{Fe}_{0.257}$ \\
\hline
\end{tabular}

The population of Fe was calculated by the difference between full population and calculated population of $\mathrm{Al}$.

proposed by Akizuki (1981). Thus, the symmetry of monoclinic vesuvianite with sectors can be explained by this mechanism. The (110) and (111) faces of vesuvianite are inclined to the $(001)$ and (100) morphological symmetry planes, resulting in the ordered structure with respect to the $\mathrm{Al}$ and $\mathrm{Fe}$ atoms. Allen and Burnham (1992) suggested this was the origin of the optical anomaly of vesuvianite as well, because the texture is similar to that of zeolites (c.g. Akizuki, 1981).

The monoclinic ordered structure is stable rather than the tetragonal disordered one at low temperature, because the tetragonal structure transforms to monoclinic in nature (Veblen and Wiechmann, 1991). The vesuvianite we have studied, which consists of both stable and metastable phases, was produced during nonequilibrium growth.

\section{Acknowledgements}

We are grateful to T. Ohyama and $\mathrm{Y}$. Ito for their assistance.

\section{References}

Akizuki, M. (1981) Origin of optical variation in analcime. American Mineralogist, 66, 403-9.

Akizuki, M. (1984) Origin of optical variation in grossular-andradite garnet. American Mineralogist, 69, 328-38.

Akizuki, M. and Sunagawa. I. (1978) Study of the sector structure in adularia by means of optical microscopy, infra-red absorption, and electron microscopy. Mineralogical Magazine, 42, 453-62.

Akizuki, M. and Terada, T. (1998) Origin of abnormal properly of apophyllite. Neues Jahrbuch fiil. Mineralogie, Monatshefte, 234-40.

Akizuki, M., Hampar, M. and Zussman, J. (1979) An explanation of anomalous optical properties of topaz. Mineralogical Magazine, 43, 237-41.

Allen, F. and Burnham, C. (1992) A comprehensive structure-model for vesuvianite: symmetry variation and crystal growth. The Canadian Mineralogist, 30. $1-18$.

Armbruster. T. and Gnos, E. (2000) P4/n and P4nc longrange ordering in low-temperature vesuvianites. American Mineralogist, 85, 563-9.

Brauns, R. (1883) Ucber die Ursache der anomalen Doppelbrechung einiger regulär krystallisirender Salze. Neues Jahrbuch für Mineralogie, Geologie und Palaeontologie, $102-11$.

Brauns, R. (1891) Die Optischen Anomalien der Kristalle. S. Hirzel, Leipzig. Germany.

Fitzgerald, S., Rheingold, A. and Leavens, P. (1986) Crystal stucture of a non-P4inne vesuvianite from Asbestos, Quebec. American Mineralogist, 71, $1483-8$.

Fitzgerald, S., Leavens, P., Rheingold, A. and Nelen, J. (1987) Crystal structure of a REE-bearing vesuvianite from San Benito County, California. American Mineralogist. 72, 625-8

Groat, L., Hawthome, F. and Ercit, T. (1992) The chemistry of vesuvianite. The Canadian Mineralogist, 30, 19-48.

Groat, L., Hawthorne, F., Ercit. T. and Putnis, A. (1993) The symmetry of vesuvianite. The Canadian Mineralogist, 31, 617-35.

Groat, L., Hawthorne, F., Rossman, G. and Lreit, T. (1995) The infrared spectroscopy of vesuvianite in the $\mathrm{OH}$ region. The Canadian Mineralogist, 33, $609-26$.

International Tables for X-ray Crystallography (1974) 
The Kynoch Press, Birmingham, England.

Johnson, T.E., Hudson, N.F.C. and Droop, G.T.R. (2000) Wollastonite-bearing assemblages from the Dalradian at Fraserburgh, northeast Scotland and their bearing on the emplacement of garnetiferous granitoid sheets. Mineralogical Magazine, 64, $1165-1176$.

Lager, G., Xie, Q., Ross, F., Rossman, G., Armbruster, T., Rotella, F. and Schultz, A. (1999) Hydrogenatom positions in $P 4 / n n c$ vesuvianite. The Canadian Mineralogist, 37, 763-8.

Mallard, E. (1877) Ueber anomale optische Erscheinungen an Krystallen. Zeitschrift für Kristallographie und Mineralogie, 309-20.

Matsubara, S., Miyawaki, R., Kato, A., Yokoyama, K. and Okamoto, A. (1998) Okayamalite, $\mathrm{Ca}_{2} \mathrm{~B}_{2} \mathrm{SiO}_{7}$, a new mineral, boron analoguc of gehlenite. Mineralogical Magazine, 62, 703-706

Molecular Structure Corporation $(1985,1992)$ teXsan: Crystal structure analysis package. Molecular Structure Corporation, Texas, USA.
Ohkawa, M., Yoshiasa, A. and Takeno, S. (1994) Structural investigation of high- and low-symmetry vesuvianite. Mineralogical Joumal, 17, 1-20.

Shtukenberg, A.G., Punin, Yu.O. and Soloviev, V.N. (2000) Effect of growth conditions on the birefringence of mixed crystals revealed in alum solid solutions. Mineralogical Magazine, 64, 837-45.

Shtukenberg, A.G., Punin, Yu.O., Frank-Kamenetskaya, O.V., Kovalev, O.G. and Sokolov, P.B. (2001) On the origin of anomalous birefringence in grandite garnets. Mineralogical Magazine, 65, 445-59.

Veblen, D. and Wiechmann, M. (1991) Domain structure of low-symmetry vesuvianite from Crestmore, California. American Mineralogist, 76, $397-404$.

Warren, B. and Modell, D. (1931) The structure of vesuvianite $\mathrm{Ca}_{10} \mathrm{Al}_{4}\left(\mathrm{Mg}, \mathrm{Fe}_{2} \mathrm{Si}_{9} \mathrm{O}_{34}(\mathrm{OH})_{4}\right.$. Zeitschrift für Kristallographie, 78, 422-32.

[Manuscript received 22 March 2001 :

revised 23 January 2002] 